\title{
MHD Mixed Convection and Entropy Generation in a C-Shaped Enclosure Filled with an Electrically Conducting Fluid
}

\author{
Rabah Bouchair ${ }^{1}$, Abderrahim Bourouis ${ }^{2, *}$ and Abdeslam Omara ${ }^{3}$ \\ ${ }^{1,3}$ Mentouri Brothers Constantine1 University, Constantine City, Algeria \\ ${ }^{2}$ Mohamad Seddik Ben Yahya University, Jijel City, Algeria \\ E-mail : bourouis2010@gmail.com
}

Received 13 January 2021, Revised 10 June 2021, Accepted 5 July 2021

\begin{abstract}
Magnetohydrodynamic (MHD) mixed convection and entropy generation in a C-shaped cavity filled by an electrically conducting fluid are investigated numerically using the finite volume method and the SIMPLE algorithm. In this work, we focus on the effect of the magnetic field on the characteristics of fluid flow, heat transfer and entropy generation for various values of Richardson number $(\mathrm{Ri}=0.1,1$ and 10), Hartmann number $(0 \leq \mathrm{Ha} \leq$ 200 ), tilting angle $(\alpha)$; ranging from $-45^{\circ}$ to $+45^{\circ}$, and aspect ratio $(A R=0.3,0.5$ and 0.7$)$. The results show an increase in the average Nusselt number and the entropy generation by increasing the aspect ratio (AR), whereas they decrease when Ha number increases, independently of $\alpha$ and $\mathrm{Ri}$. At high values of Ha number, the conduction state is the dominant mode of heat transfer regardless of $\mathrm{Ri}, \mathrm{AR}$ and $\alpha$. Moreover, the total entropy generation is mainly due to the irreversibility of heat transfer whatever the control parameters are.
\end{abstract}

Keywords: Mixed convection; C-shaped enclosure; magneto-hydrodynamic; electrically conducting fluid; finite volume method.

\section{Introduction}

It is well known that traditional coolants such as air, water and ethylene glycol are characterized by low thermal conductivity which affects the rate of heat transfer in confined structures. To overcome this limitation, natural or mixed convection inside cavities with various shapes including square, rectangular, trapezoidal, $\mathrm{C}, \mathrm{L}, \mathrm{U}, \mathrm{F}$ or T have been studied by many researchers due to their implication in many technological applications, such as heat generating components, home ventilation, micro-channel, solar energy industry, etc. [1, 2]. Besides, it is found that the enclosure shape can have and important impact on the improvement heat transfer rate in these engineering devices.

The effect of magnetic field on convection of electrically conducting fluids inside cavities with various shapes has been thoroughly studied by many researchers. In such a situation, the application of an external magnetic field results in damping the flow and temperature oscillations what is desired in several industrial processes and applications such as crystal growth.

The heat transfer process in real thermal system is obviously an irreversible phenomenon. Such irreversibility is synonymous with loss of energy or dissipation due to friction and intermolecular collisions, not recoverable if the process is reversed, which is also synonymous with destroyed exergy. As a result, a reduction of the overall energy efficiency of the system is observed. Thus, in order to quantify this energy loss and in the same way, control the reduction of the overall energy efficiency of the system, we use the second law of thermodynamics by studying entropy generation which is the basis of most formulations for systems under equilibrium or non-equilibrium thermodynamics state.

In fact, in the natural convection process, the loss of the available energy is due to heat transfer and friction of fluids. Thus, by knowing which of the two previous components destroy the most exergy, the efficiency of the thermal system can be improved by setting the optimal value of the controlling parameters, which results in a minimization of entropy generation.

In this context, many works have been published on this topic. In order to simulate heat transfer and fluid flow during crystal growth, Oreper and Szekely [3] studied the effect of the gravity and magnetic fields on an electrically conducting liquid in a rectangular cavity. They reported that the reduction in the absolute value of the velocity increases with the strength of the imposed magnetic field. Unfortunately, due to the fact that the maximum attainable intensity of the magnetic field is of the order of a few thousand gauss, the movement of the fluid cannot be completely suppressed. Natural convection of an electrically conducting fluid, equivalent to melt silicium ( $\mathrm{Pr}$ $=0.054)$, in a square cavity under both magnetic and gravitational field has been investigated numerically by Ozoe and Maruo [4]. Their numerical results reveal that at $\mathrm{Ha}=10^{3}$ and $\mathrm{Ra}=10^{6}$, the temperature profile is almost linear indicating an almost conductive state. Garandet et al. [5] studied the effect of a transverse magnetic field on buoyancy driven convection in a two-dimensional cavity using analytical approaches. They observed that within the limit of the high Hartmann numbers, the velocity gradient in the flow core is constant except in the two Hartmann 
layers in the vicinity of the walls normal to the magnetic field.

Experimental measurement of heat transfer rates by natural convection of molten gallium $(\operatorname{Pr}=0.024)$ with and without external magnetic field in a cubic enclosure has been carried out by Okada and Ozoe [6]. They found that a much higher suppression effect is observed when the magnetic field is perpendicular to the vertical hot wall ( $\mathrm{x}$ direction) or vertical ( $\mathrm{z}$ direction) than that when the magnetic field is parallel and horizontal to the hot wall (y direction). Emery [7] investigates the effect of a magnetic field on heat transferred by natural convection of water and a conducting fluid (mercury) through vertical plane layers. Venkatachalappa and Subbaraya [8] have examined numerically MHD free convection of an electrically conducting fluid in a two-dimensional rectangular enclosure in which the two side walls are maintained at uniform heat flux condition, while the horizontal top and bottom walls are thermally insulated. They concluded that for sufficiently high magnetic field strength, the convection is suppressed regardless of Grashof number. The effect of a transverse magnetic field on buoyancy-driven convection in a shallow rectangular cavity has been studied numerically by Alchaara et al. [9]. In their study both cases of a cavity with all rigid boundaries and a cavity with a free upper surface are considered. Based on parallel flow approximation, the authors have made a comparison with an analytical solution. Their results show good agreement between the numerical and the analytical results was observed when the aspect ratio of the cavity is equal to or greater than approximately three and for a given range of the governing parameters ( $\mathrm{Ra}, \mathrm{Ha}, \mathrm{Pr}$ ) for which the effect of convective heat transfer is negligible.

Rudraiah et al. [10] studied numerically, using ADI method to solve the vorticity-stream function formulation, the magneto-hydrodynamique natural convection inside a rectangular cavity filled with an electrically conducting fluid. They have found that for high $\mathrm{Gr}$ and low $\mathrm{Ha}$ numbers, convection is the dominant heat transfer mechanism leading to vertical temperature stratification in the core region. On the contrary, for sufficiently large Hartmann number the temperature stratification in the core diminishes and the thermal boundary layers at the two side walls disappear. Al-Najem et al. [11] have examined the influence of external magnetic field on the heat transfer process inside tilted enclosures for a wide range of inclination angles at moderate and high Grashof numbers. Their results indicate that the inclination angle of cavity and the strength of magnetic field affect considerably the heat transfer rate and the fluid flow. Ece and Büyük [12] consider the natural convection in an inclined rectangular cavity heated and cooled on adjacent walls in the presence of a magnetic field. They have examined the effect of Grashof and Hartmann numbers, aspect ratio, angle of inclination and magnetic field direction. The effect of magnetic field on natural convection in a square cavity is considered by Sathiyamoorthy and Chamkha [13-14] and Bhuvaneswari et al. [15]. They considered non uniform heated walls such as: linear or sinusoidal temperature variation. Generally, they found that the rate of heat transfer reduces as the strength of magnetic field increases.

Although many works have been carried out to study the effect of magnetic field on natural convection, most of them have considered square or rectangular cavities. Recently, researchers are considering other geometric shapes of cavities such as trapezoidal (Hasanuzzaman et al. [16], Hossain and Abdul Alim [17]), curved shape (Sheikholeslami et al. [18]), T shape (Sahi et al. [19]), and L shape (Jahanbakhshi et al. [20]). The numerical results for wide range of Hartmann, Prandtl and Rayleigh numbers, inclination angle and for both horizontal and vertical magnetic field directions are discussed in terms of velocity, temperature field, streamlines, isotherms and local and average Nusselt number.

Mohamed and Sezai [21] have considered a Trombe wall by analyzing heat transfer by natural convection for a $\mathrm{C}$-shaped thermosyphon. The results were presented for various values of Rayleigh number and the aspect ratio of the cavity. Izadi et al. [22] numerically treated natural convection inside a $\mathrm{C}$-shaped cavity filled by a nanofluid using Lattice Boltzmann method. The obtained results showed the effect of the aspect ratio of the cavity, aspect ratio of the heat source and Rayleigh number on the fluid flow and heat transfer. They reported that the effect of the aspect ratio of the heat source on the average Nusselt number decreases at higher values of the Rayleigh number. Mansour et al. [23] studied numerically, using the finite difference method, natural convection inside $\mathrm{C}$-shaped enclosures filled with $\mathrm{Cu}$-water nanofluid. They have found, as expected, that the increase in the Rayleigh number intensify the natural convection flow, resulting in a reduction of the heat source temperature. Moreover, the increase of the solid volume fraction of nanoparticles also contributes to lowering the maximum temperature of the heat source, in particular at low Rayleigh numbers where conduction dominates the heat transfer. Yadollahi et al. [24] investigated the natural convection heat transfer performance of silver-water nanofluid with MHD effects in an F-shaped cavity. The results were presented for various values of the Rayleigh number, the Hartmann number, the aspect ratio of the F-shaped cavity, and the solid volume fraction of nanoparticles. Ghasemi [25] made a numerical study on laminar natural convection in a U-shaped enclosure filled with a water-Cu nanofluid subjected to a horizontal magnetic field. It has been shown that the increase of Rayleigh number and the solid volume fraction results in an increase of the heat transfer rate. It is found also that the increase of the heat transfer rate due to nanofluid compared to pure water is more appreciable when conduction is the dominant mode of heat transfer. Bayareh et al. [26] studied mixed convection heat transfer of alumina-water nanofluid in an inclined and baffled C-shape enclosure. They found that at high Reynolds number, increase of nanoparticles has less effect on the heat transfer rate than low ones and that heat transfer increases with the Richardson number, the enclosure angle and the length of baffle.

Indeed, many studies have taken into account, during the analysis of thermal engineering problems, the energy losses caused by heat transfer and friction of fluid which result in the entropy generation. Such analysis is useful in optimizing energy by minimizing the generation of entropy and thus, maximizing the efficiency and performance of the system. In this context, Shavik et al. [27] studied the entropy generation from natural convection inside an inclined square cavity with differentially heated vertical walls. They found that with increase of Rayleigh number, an increase of the entropy generation due to fluid friction and a decrease of the average Bejan number are observed. Also, their results showed that as the inclination angle 
increases the variation of heat transfer irreversibility is found to be small while the corresponding one to fluid friction inside the cavity varies considerably. Parvin and Chamka [28] analyzed numerically the natural convective heat transfer and entropy generation inside an odd-shaped cavity filled with either water or $\mathrm{Cu}$-water nanofluid. Results are presented for various Rayleigh numbers and solid volume fractions. They found that by increasing Rayleigh number, a decrease of the viscous term and an enhancement of the entropy generation due to heat transfer are observed. They also showed that a judicious choice of the Rayleigh number will allow us to maximize the rate of heat transfer and minimize the generation of entropy. Chamkha et al. [29] investigated numericallay MHD mixed coonvection and entropy generation in a lid-driven traingular cavity filled by nanofluid. The effect of Richardson and Hartmann numbers on the convective heat transfer and entropy generation for different electrical conductivity models of nanofluid is examined. Cho [30] also investigated the problem of mixed convection heat transfer and entropy generation in a lid-driven wavy-wall cavity filled with $\mathrm{Cu}$-water nanofluid. The results showed that both the average Nusselt number and total entropy generation increase as the amplitude of the wavy surface increases. It is found also that, depending on the Richardson numbers, an optimal heat transfer is obtained for a given appropriate wavelength of the wavy surface. Khakrah et al. [31] analyzed free convection heat transfer performance of a sinewalled cavity filled with filled with $\mathrm{Al}_{2} \mathrm{O}_{3}$-water nanofluid using the compact finite-difference lattice Boltzmann methode. They demonstrated that the total entropy generation reduces with adding nanoparticles to the base fluid, while it has a direct relationship with the Rayleigh number.

In the light of the above literature review, we notice that the geometry of a C-shaped cavity has not received many interests in the literature both in the case with natural or mixed convection with or without MHD effect. Thus, motivated by the previous studies, the present study considers a numerical solution for mixed convection fluid flow and entropy generation of an electrically conducting fluid inside $\mathrm{C}$-shaped enclosure. Through the numerical results, we try to present a detailed understanding of the effects of the Richardson number (Ri), Hartmann number (Ha), the tilting angle $(\alpha)$ as well as the aspect ratio (AR) on the flow structure, the heat transfer characteristics, entropy generation and Bejan number.

\section{Geometry and Mathematical Formulation}

The geometry of the considered physical model is schematically demonstrated in Figure 1. It is a two dimensional cavity of length $L$ and high $H$, where the insides walls $(A B, B C$ and $C D)$ have an aspect ratio $A R=$ $l / L$ (where $l=L_{x}=0.5$ or $l=L_{y}=0.5$ ). It is assumed that the two horizontal walls of the cavity are thermally insulated, and that the C-shaped wall and the right vertical movable wall are maintained at constant different temperatures, $\theta_{h}$ and $\theta_{c}$ respectively. The mixed convection of an electrically conducting fluid $(\mathrm{Pr}=7)$ inside the $\mathrm{C}$ shaped enclosure is subjected to a horizontal external uniform magnetic field. Some assumptions are considered in this study to simplify the numerical computations by taking into consideration that the fluid flow inside the cavity is steady, two dimensional, laminar and incompressible except for the buoyancy term where the
Boussinesq approximation is assumed to be valid. The physical properties of the fluid are supposed constant. It is also assumed that the other heat generation sources, such as viscous dissipation, Joule heating effect and the induced magnetic field are negligible.

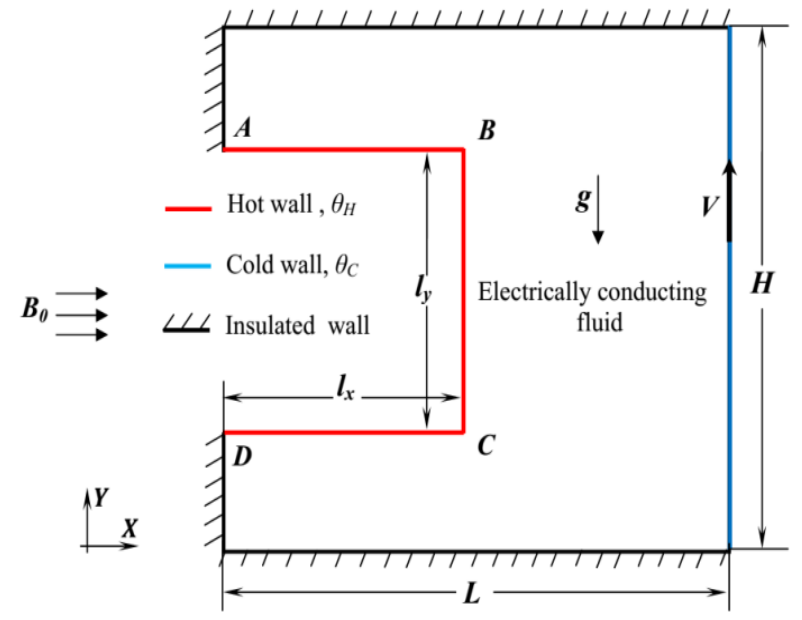

Figure 1. Schematic diagram of the C-shaped enclosure with related boundary conditions.

Given the above assumptions, the governing equations under the effect of magnetic field can be written in dimensionless form using the following dimensionless variables:

$$
\begin{aligned}
& X=\frac{x}{L}, Y=\frac{y}{H}, U=\frac{u}{U_{0}}, V=\frac{v}{U_{0}}, \theta=\frac{T-T c}{\Delta T}, P=\frac{p}{\rho U_{0}^{2}} \\
& \frac{\partial U}{\partial X}+\frac{\partial V}{\partial Y}=0 \\
& U \frac{\partial U}{\partial X}+V \frac{\partial U}{\partial Y}=-\frac{\partial P}{\partial X}+\frac{1}{\operatorname{Re}}\left[\frac{\partial^{2} U}{\partial X^{2}}+\frac{\partial^{2} U}{\partial Y^{2}}\right] \\
& U \frac{\partial V}{\partial X}+V \frac{\partial V}{\partial Y}=-\frac{\partial P}{\partial Y}+\frac{1}{\operatorname{Re}}\left[\frac{\partial^{2} V}{\partial X^{2}}+\frac{\partial^{2} V}{\partial Y^{2}}\right]-\frac{H a^{2}}{\operatorname{Re}}+R i \theta \\
& U \frac{\partial \theta}{\partial X}+V \frac{\partial \theta}{\partial Y}=\frac{1}{\operatorname{Re} \operatorname{Pr}}\left[\frac{\partial^{2} \theta}{\partial X^{2}}+\frac{\partial^{2} \theta}{\partial Y^{2}}\right]
\end{aligned}
$$

Where: $R i, G r, R e, P r$ and $H a$ are the Richardson, Grashof, Reynolds, Prandtl and Hartmann numbers, respectively and are defined below:

$$
\begin{aligned}
& R i=G r / \operatorname{Re}^{2}, G r=g \beta\left(T_{H}-T_{c}\right) H^{3} / v^{2}, \operatorname{Re}=U_{0} H / v \\
& \operatorname{Pr}=v / \alpha_{f}, H a=B_{0} H \sqrt{\sigma / \rho v}
\end{aligned}
$$

The local entropy generation of the system represents the irreversibility due to thermal gradient, fluid friction and the magnetic force is defined as follows:

$$
S_{L}=S_{L, T h}+S_{L, V i s c}+S_{L, M a g}
$$

Where

$$
\begin{aligned}
& S_{L, T h}=\left(\frac{\partial \theta}{\partial X}\right)^{2}+\left(\frac{\partial \theta}{\partial Y}\right)^{2} \\
& S_{L, V i s c}=\chi_{1}\left[2\left(\frac{\partial U}{\partial X}\right)^{2}+2\left(\frac{\partial V}{\partial Y}\right)^{2}+\left(\frac{\partial U}{\partial Y}+\frac{\partial V}{\partial X}\right)^{2}\right] \\
& S_{L, \text { Mag }}=\chi_{2}(U \sin \alpha-V \cos \alpha)^{2}
\end{aligned}
$$


The irreversibility distribution ratio due to viscous effect and the irreversibility coefficient due to magnetic field effect are defined, respectively by:

$$
\begin{aligned}
& \chi_{1}=\frac{\mu T_{0}}{k}\left(\frac{v}{L(\Delta T)}\right)^{2} \\
& \chi_{2}=\chi_{1} H a^{2}
\end{aligned}
$$

The total dimensionless entropy generation is calculated by integrating the local entropy generation (Eq. 6) through the entire cavity and is given by:

$$
S_{T}=\int_{V} S_{L} d V
$$

We also define the average Bejan number $\left(B e_{a v g}\right)$ which indicates which irreversibility mode, heat transfer or fluid friction, dominates the total entropy generation.

$$
B e_{a v g}=\frac{S_{T, T h}}{S_{T}}
$$

Thus: if $B e_{\text {avg }}>0.5$ heat transfer irreversibility (HTI) dominates, while fluid friction irreversibility (FFI) dominates when $B e_{a v g}<0.5$, and finally when $\cong 0.5$ corresponds to the case where, HTI and FFI are of equal importance.

The associated dimensionless form of the boundary conditions can be written as follows:

On C-shaped wall:

$$
\left\{\begin{array}{l}
\text { Side } A B: U=V=0, \quad \theta=1, \quad 0 \leq X \leq l_{x}, \quad Y=\frac{l_{y}}{2} \\
\text { Side } D C: U=V=0, \quad \theta=1, \quad 0 \leq X \leq l_{x}, \quad Y=l_{y}+\frac{l_{y}}{2} \\
\text { Side } B C: U=V=0, \quad \theta=1, \quad X=l_{x}, \quad \frac{l_{y}}{2} \leq Y \leq l_{y}+\frac{l_{y}}{2}
\end{array}\right.
$$

On right wall

$$
U=0, V=1, \theta=0, \quad X=1,0 \leq Y \leq H
$$

On insulated walls

$$
\begin{cases}U=V=\frac{\partial \theta}{\partial X}=0, & X=0,0 \leq Y \leq \frac{l_{y}}{2} \text { and } l_{y}+\frac{l_{y}}{2} \leq Y \leq 1 \\ U=V=\frac{\partial \theta}{\partial Y}=0, & 0 \leq X \leq 1, Y=0 \\ U=V=\frac{\partial \theta}{\partial Y}=0, & 0 \leq X \leq 1, Y=1\end{cases}
$$

The local Nusselt number at the isothermal walls can be defined by the following equations:

- On the hot $\mathrm{C}$-shaped walls ( $A B, B C$ and $C D$ sides)

$$
\begin{array}{ll}
\text { a) Side }(A B) & N u_{L}=-(\partial \theta / \partial Y)_{Y=l_{y}+\frac{l_{y}}{2}} \\
\text { b) Side }(B C) & N u_{L}=-(\partial \theta / \partial X)_{X=l_{X}} \\
\text { c) Side }(C D) & N u_{L}=-(\partial \theta / \partial Y)_{Y=l_{y}}
\end{array}
$$

- On the vertical cold wall

$$
N u_{L}=-(\partial \theta / \partial X)_{X=L}
$$

The total average Nusselt number is determined by integrating the local Nusselt number along the hot C-shaped wall and the vertical cold wall as follows:

- On the hot C-shaped wall

$$
N u_{\text {avg }}=\left.\int_{B}^{A} N u_{L} d X\right|_{Y=l_{y}+\frac{l_{y}}{2}}+\left.\int_{C}^{B} N u_{L} d Y\right|_{X=l_{X}}+\left.\int_{D}^{C} N u_{L} d X\right|_{y=l_{y}}
$$

- On the vertical cold wall

$$
N u_{\text {avg }}=\left.\int_{0}^{H} N u_{L} d Y\right|_{X=H}
$$

\section{Numerical Method}

The set of eqs. (2-5) governing magnetohydrodynamic mixed convection along with the corresponding boundary conditions are discretized using the finite volume method, and the power-law scheme is used for the convection diffusion terms as given by Patankar [32]. Besides, the control volume discretization provides the continuity of the convective and diffusive fluxes. A non-uniform grid is used for the numerical computations, and the considered non uniform mesh is shown in Figure 2. Numerical solution is assured by an iterative method using the SIMPLE algorithm to treat the high coupling between the pressure and velocity. The line by line iterative method and the tri-diagonal matrix algorithm (TDMA) are used to resolve the resulting discretized algebraic equations. This solution procedure is repeated until the difference, in absolute value, between two successive solutions of velocity and temperature at each mesh point is less than $10^{-5}$. In addition, the difference of the amount of heat passes through the hot and cold walls should be conserved and this difference is assumed to be less than $10^{-3}$ (i.e., $N u_{\text {avg }}$ (at cold wall) - $N u_{\text {avg }}$ (at hot wall) < $\left.10^{-3}\right)$. All numerical computations are obtained using an inhouse FORTRAN code developed by the authors.

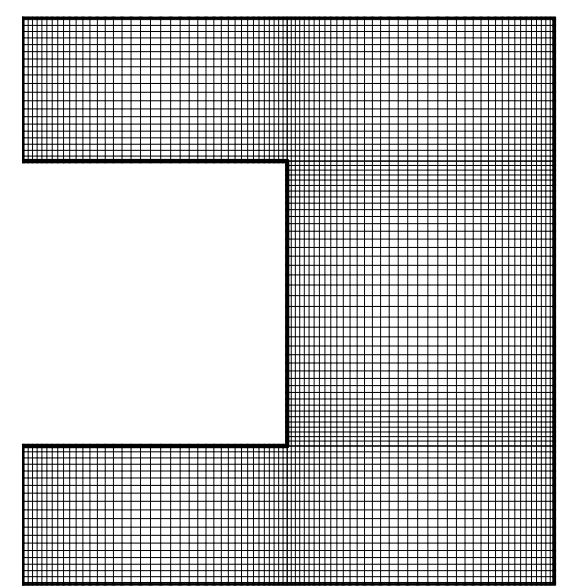

Figure 2. Non-uniform mesh used in all numerical computations.

A grid-independence study is performed by considering several number of control volumes. Six cases are tested ranging from coarse grid of $22 \times 22$ to much finer grid of $122 \times 122$ control volumes of dimension $\Delta X \times \Delta Y$ (Figure 2). The variation of the average Nusselt number as a function of the above chosen grid sizes at $R a=100, H a=0, A R=$ 0.5 and $\alpha=0$ and for three values of Richardson number $(R i=0.1,1$ and 10) is shown in Table 1. It is clearly observed that beyond a grid size of $82 \times 82$ control volumes 
ensure largely the grid-independence numerical solution. Thus, by taking into account the required precision and the calculation time, the grid size of $82 \times 82$ control volumes is chosen for all the calculations reported in this study. It should be noted that the grid used in the present numerical simulation is divided into two regions in the $X$ direction and three regions in $Y$ direction in order to ensure a refined mesh near the active walls where the gradients are more important.

Table 1. Mesh sizes effect for different values of Ri number.

\begin{tabular}{cccccccc}
\hline & \multicolumn{3}{c}{$R i=0.1$} & \multicolumn{2}{c}{$R i=1$} & \multicolumn{2}{c}{$R i=10$} \\
\hline Grid Size & $N u_{\text {avg }}$ & $\begin{array}{c}\text { Error } \\
(\%)\end{array}$ & $N u_{\text {avg }}$ & $\begin{array}{c}\text { Error } \\
(\%)\end{array}$ & $N u_{\text {avg }}$ & $\begin{array}{c}\text { Error } \\
(\%)\end{array}$ \\
\hline $22 \times 22$ & 7.098687 & 1.2817 & 6.058383 & 2.1748 & 6.645239 & 3.5345 \\
$42 \times 42$ & 7.189679 & 0.5940 & 5.926620 & 2.3677 & 6.410358 & 1.8148 \\
$62 \times 62$ & 7.146966 & 0.2107 & 5.786294 & 1.4406 & 6.294022 & 1.2193 \\
$82 \times 82$ & 7.131905 & 0.2049 & 5.702934 & 1.0271 & 6.217274 & 0.7415 \\
$102 \times 102$ & 7.117291 & 0.1503 & 5.644357 & 0.7016 & 6.171171 & 0.5408 \\
$122 \times 122$ & 7.106593 & - & 5.604754 & - & 6.137796 & - \\
\hline
\end{tabular}
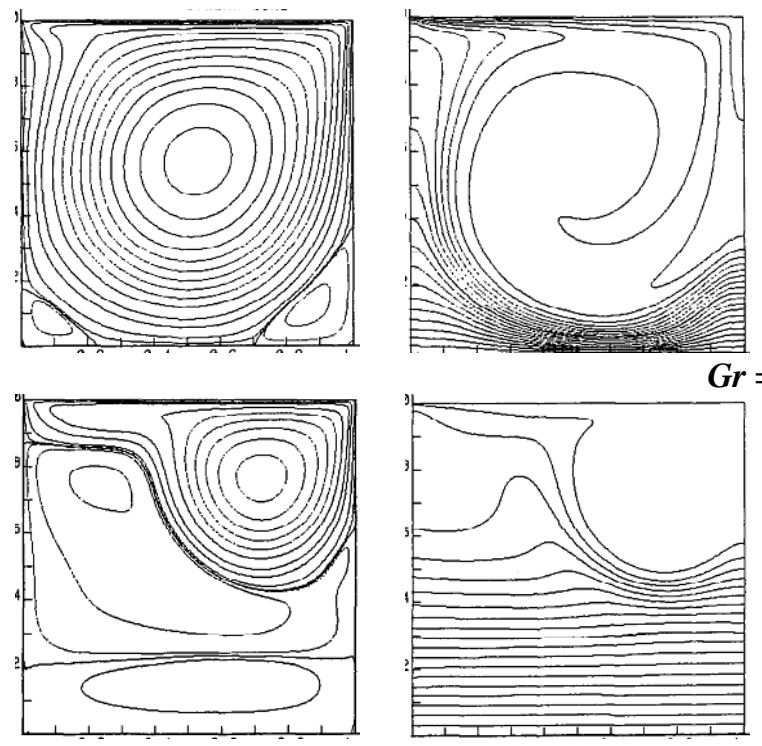

Results of Iwatsu et al.[31]
Besides, the accuracy of our numerical results is verified by performing two validations under special cases with the results available in the literature. The first validation is performed by comparing our numerical results to those obtained Iwatsu et al. [33] for the case of mixed convection in a cavity filled with air under vertical temperature gradient at two values of Grashof number $(\mathrm{Gr}$ $=10^{2}$ and $\left.10^{6}\right), \operatorname{Pr}=0.71$, and $R e=1000$. As can be shown by comparing of the streamlines and isotherms in Figure 3, the good agreement between the two sets of results is observed.

The second validation concerns the comparison of the results obtained by the developed computational FORTRAN code for natural convection under the effect of magnetic field in a C-shaped cavity filled with ferrofluid liquid with those obtained by Mojumder et al. [34] in the case of clear fluid (i.e., $\varphi=0$ ) at $R a=10^{3}$ and $H a=25$. The results from the in-house FORTRAN code also show excellent agreement as can be seen in Figure 4.
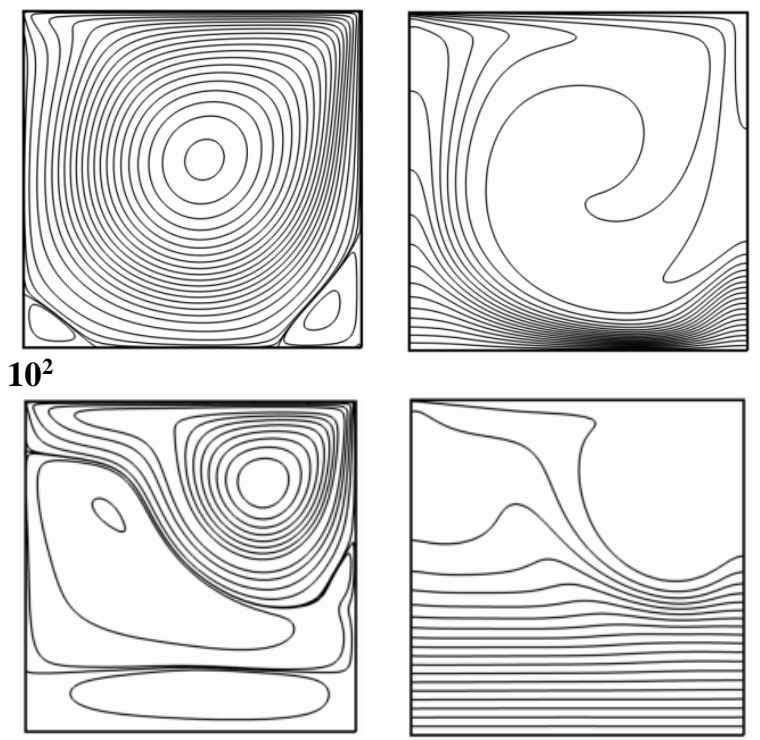

$G r=10^{6}$

Our results

Figure 3. Comparison of streamlines and isotherms between our results (on the right) and that of Iwatsu et al. [33] (on the left) at $\mathrm{Gr}=102$ and 106, $\mathrm{Pr}=0.71, \mathrm{Re}=1000$.

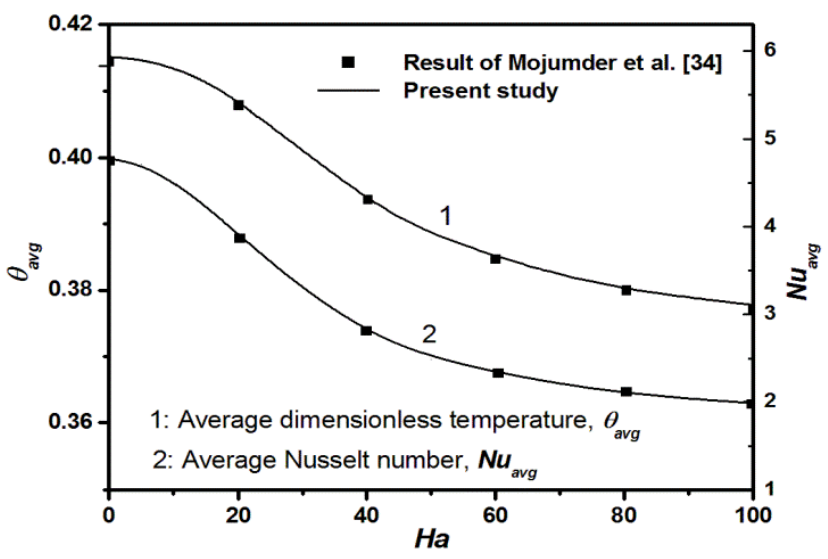

Figure 4. Comparison of the average dimensionless temperature and the average Nusselt number a function of $\mathrm{Ha}$ number of the case of clear fluid with the results of Mojumder et al. [34].
It can be concluded that these two favorable comparisons ensures the reliability of the in-house FORTRAN code for studying mixed convection inside a Cshaped enclosure.

\section{Result and Discussion}

In this section, the results of a numerical study on the MHD mixed convection and entropy generation in a Cshaped cavity filled by an electrically conducting fluid are discussed. The effect of various controlling parameters, such as the Richardson number $(0.1 \leq R i \leq 10)$, the Hartmann number $(0 \leq H a \leq 200)$, the tilting angle of the cavity $\left(-45^{\circ} \leq \alpha \leq+45^{\circ}\right)$ and the aspect ratio $(A R=0.3,0.5$ and 0.7 ) on the flow structure and thermal field, and on the heat transfer rate is examined. In this study, the Prandtl number $(P r)$, the Reynolds number $(R e)$, and the irreversibility fluid friction coefficient $\left(x_{l}\right)$ are fixed at 7 , 100 and $10^{-3}$, respectively. 


\subsection{Effect of Hartmann and Richardson numbers}

Figures 5-7 show the effect of Hartmann number on the flow pattern, temperature distribution and isentropic lines for three various values of Richardson number: $R i=0.1$ (forced convection), 1.0 (mixed convection), and 10 (natural convection) at $\alpha=0^{\circ}$ and $A R=0.5$.

Figure 5 illustrates the effect of increase in $\mathrm{Ha}$ number on the streamlines, isotherms and isentropic lines inside the cavity for the case of forced convection dominated regime $(R i=0.1)$. In the absence of magnetic field $(\mathrm{Ha}=0)$, the flow pattern is characterized by a main anti-clockwise cell generated by the moving wall and two secondary small clockwise cells, located at the left bottom and the left top corners, generated by the buoyancy force. The isotherms show a significant distortion near the cold wall of the cavity due to the effect of the moving wall. At the top edge of the vertical hot wall $(B C)$, the isotherms are denser resulting in an increase in the temperature gradient and therefore the heat transfer rate at this location. As shown by the isentropic lines, the local entropy generation is limited around the point $\mathrm{B}$, the hot side $B C$ and the bottom edge of cold wall. Elsewhere, no significant local entropy generation is observed.
As $H a$ is increased to 25 , the strength of the fluid circulation decreases and the streamlines become denser in the vicinity of the moving lid as a consequence of the electromagnetic force effect that acts in the opposite direction of the fluid flow. The isotherms become less distorted and less clustered at the top edge of the vertical hot wall which results in a decrease the heat transfer rate. The corresponding isentropic lines become less dense leading to a decrease in the local entropy generation. By increasing $\mathrm{Ha}$ up to 100 , the two secondary small cells disappear as a result of a high intensity of magnetic field and low value of $R i$ number. Consequently, the flow pattern shows a single anticlockwise cell, generated by moving lid, with significant reduction of stream function value. As a result, the fluid at the corner of the cavity becomes stagnant. The isotherms show a nearly parallel distribution indicating that the heat transfer is predominantly done by conduction. A further decrease of local entropy generation in the above mentioned regions is observed, which indicates a decrease of thermal gradient and viscous friction irreversibility under the influence of the magnetic field.
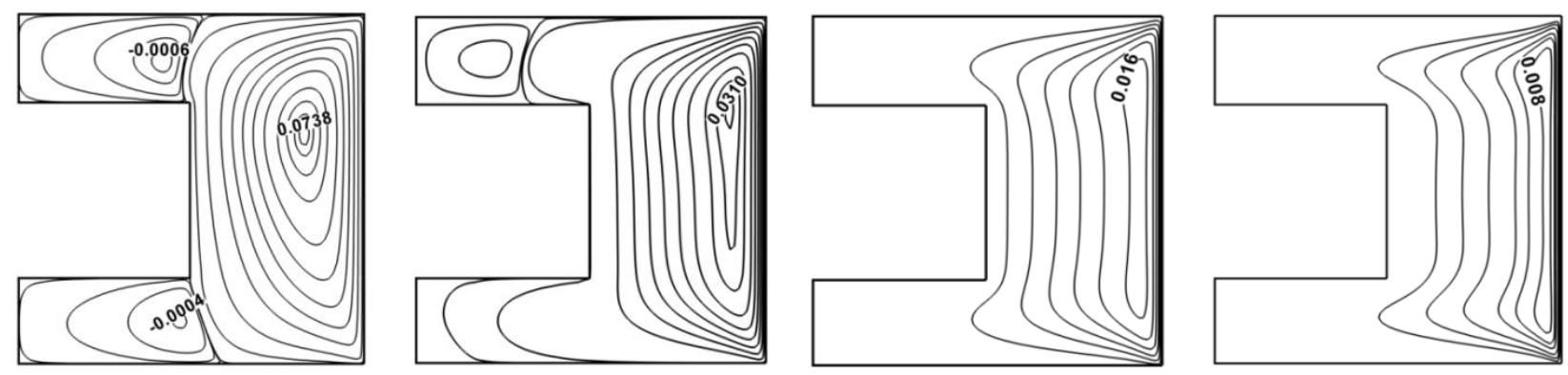

Streamlines
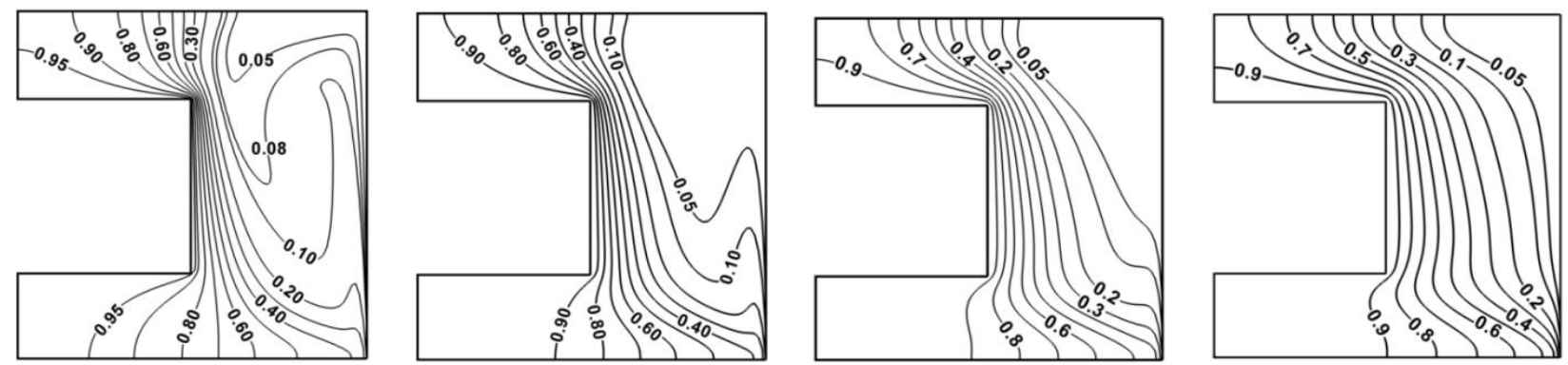

Isotherms
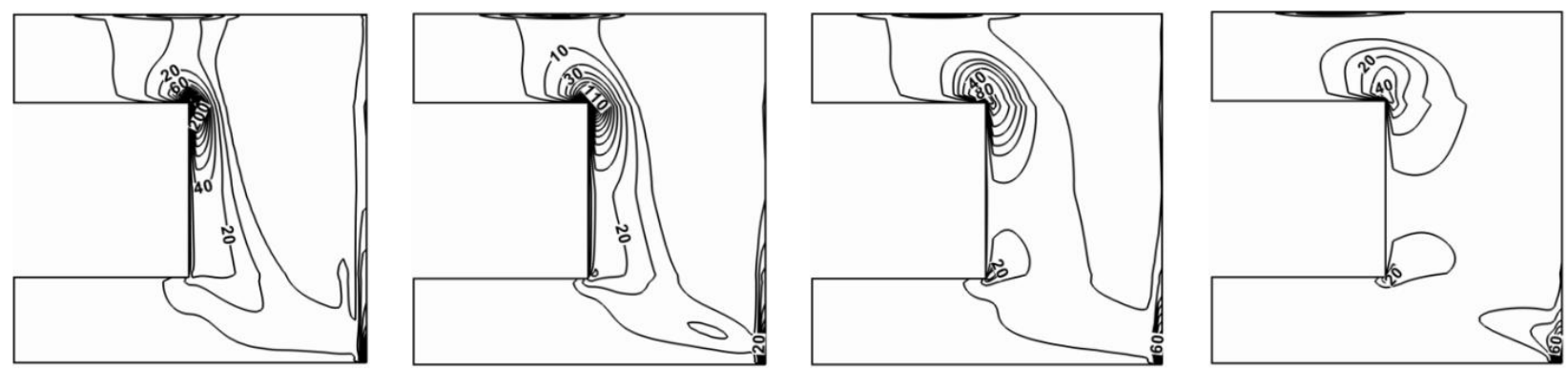

Isentropic lines

$H a=0$

$H a=25$

$H a=50$

$H a=100$

Figure 5. Streamlines, isotherms and isentropic lines for various Ha number at Ri $=0.1$.

As $R i$ is increased to 1 , the flow remains multi-cellular. However, a slight increase of the intensity and the size of the secondary cells to the detriment of the cell generated by moving lid (anticlockwise cell) are observed when $\mathrm{Ha}=0$, Figure 6. The corresponding isotherms and isentropic lines have almost the same distribution as that corresponding to the case where $R i=0.1$. An almost similar behavior of streamlines, isotherms and isentropic lines to the case of Figure $5(R i=0.1)$ is observed by increasing the $\mathrm{Ha}$ 
number from 0 to 100 , except the fact that, in this case, the main counterclockwise cell splits into two parts.

Figure 7 presents the flow patterns, the isotherms and isentropic lines for various $\mathrm{Ha}$ number in the case where natural convection is dominant (i.e., $R i=10$ ). It can be observed that the flow pattern remains bi-cellular regardless of $H a$ number values. Besides, at $H a=0$, due to the strong buoyancy force, the two small cells, observed in Figures 5 and 6 , merge to form a large clockwise rotating cell, leading to weakening the intensity and the extent of the counterclockwise cell (generated by the movable wall).

Though a progressive decrease of the flow intensity inside the cavity is observed by increasing $\mathrm{Ha}$ from $\mathrm{O}$ to 100, a slight increase of the extent of both cells is observed. The deformation of the corresponding isotherms appears to be significantly reduced by increasing the $H a$ number. At $H a=100$, the isotherms become almost parallel to the vertical walls indicating that the heat transfer is primarily by conduction. The local entropy lines are concentrated around the point $\mathrm{C}$ and the right corner of top wall at $\mathrm{Ha}=$ $O$ as can be seen in Figure 7. As $\mathrm{Ha}$ increases up to 100, the density of the entropy lines is decreased. Hence, a decrease of local entropy generation is observed as a result of the increase of the Lorentz force.

In Figure 8, the effect of $H a$ number on the local Nusselt number along the hot wall (i.e., $A B C D$ ) and on along the cold right wall of the cavity is presented. Recall that the values of the $R i$ number remain the same as above, i.e. $R i=0.1,1$ and 10 .
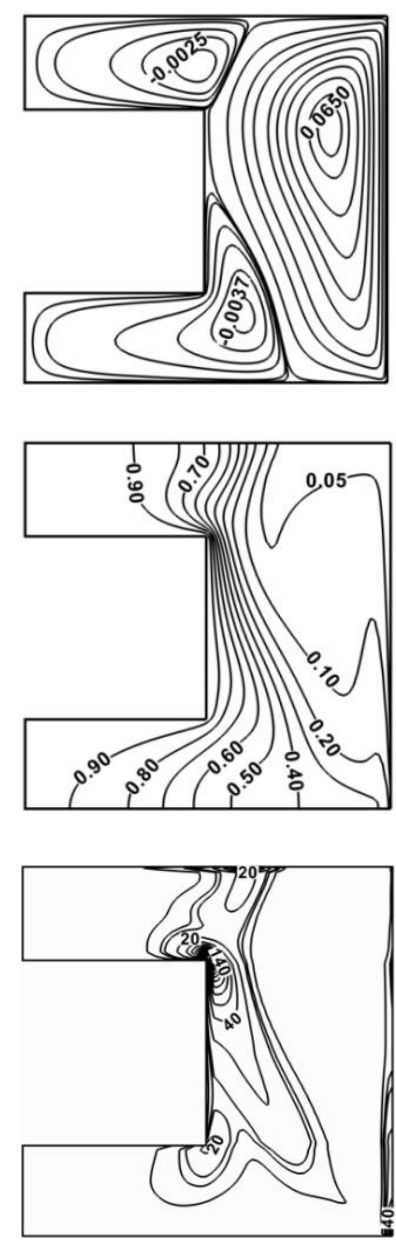

$H a=0$
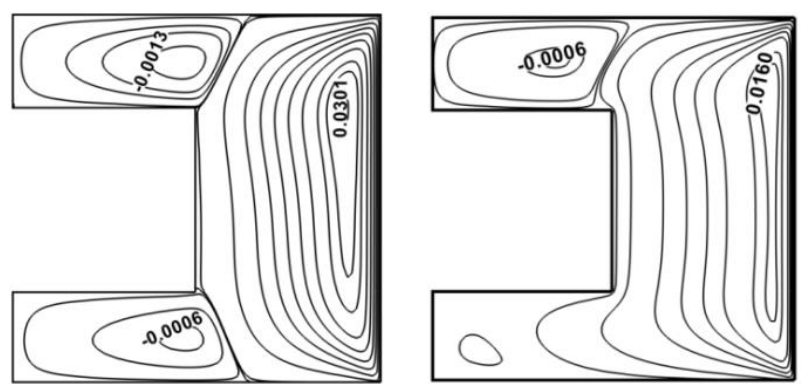

Streamlines
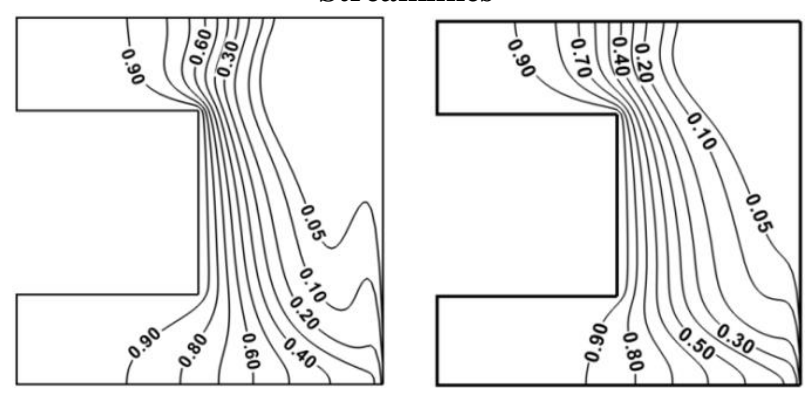

Isotherms
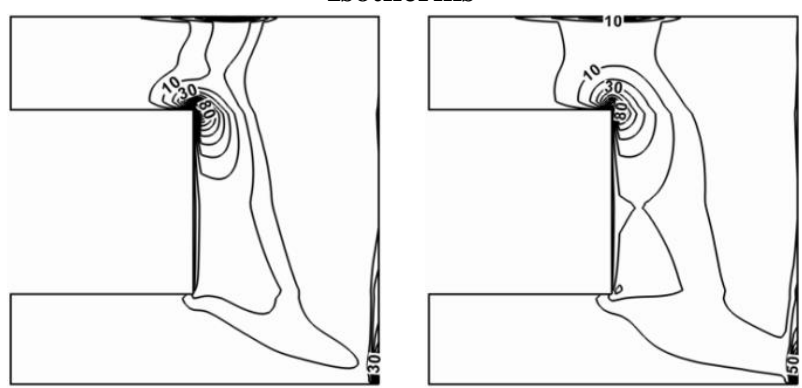

Isentropic lines

$$
H a=25 \quad H a=50
$$
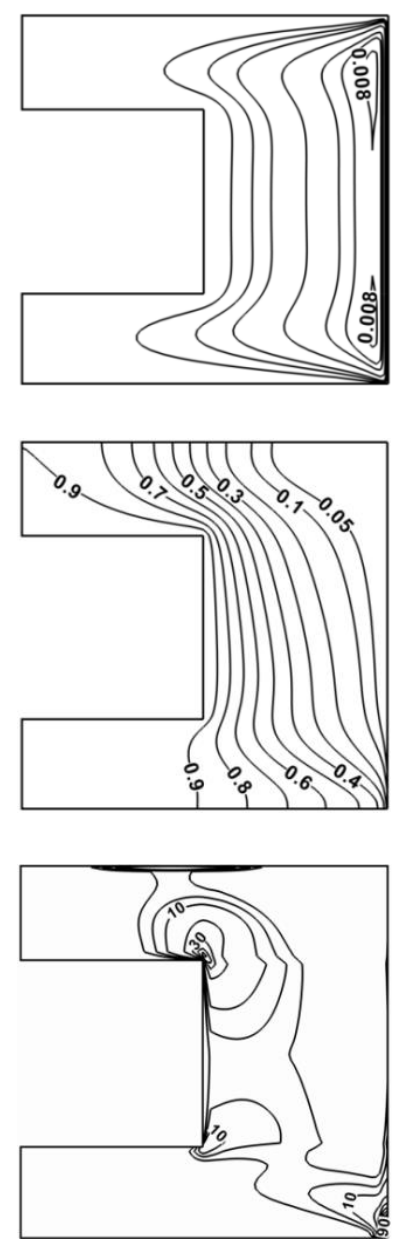

$\mathrm{Ha}=100$

Figure 6. Streamlines, isotherms and isentropic lines for various Ha number at Ri $=1$. 


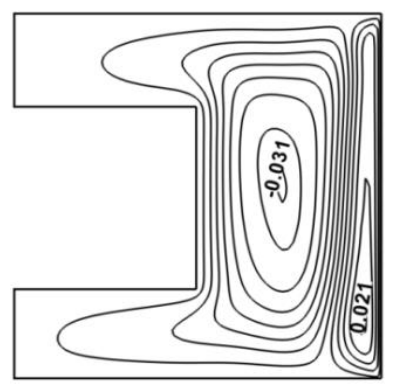

$H a=0$
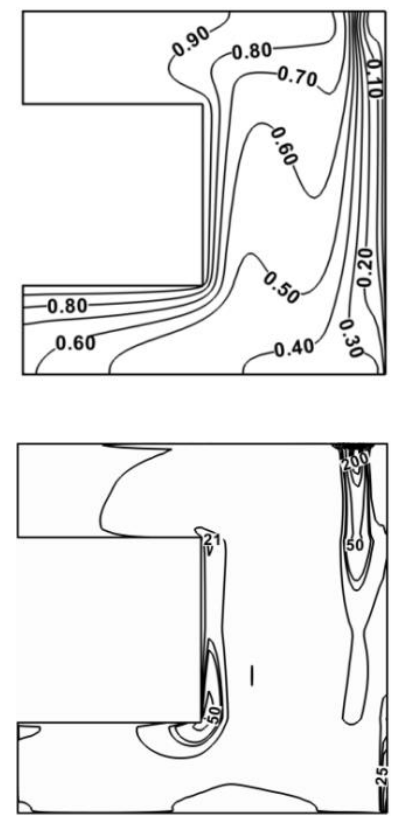

$H a=0$
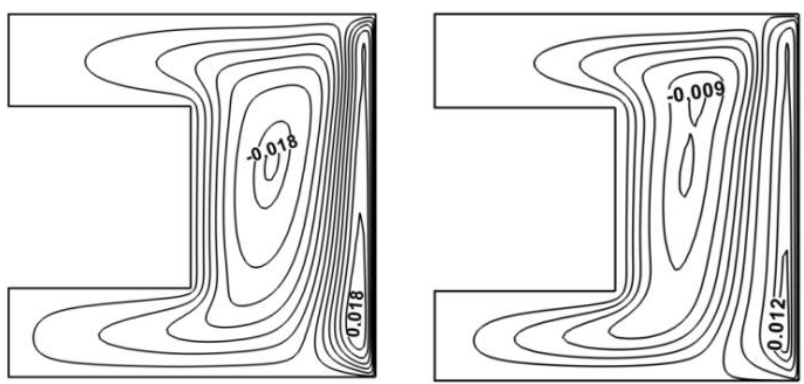

Streamlines

$H a=25$

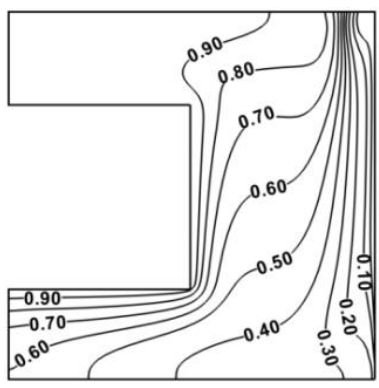

$H a=50$

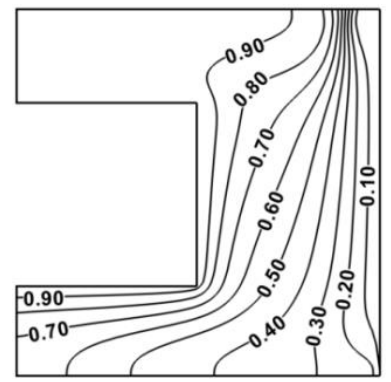

Isotherms

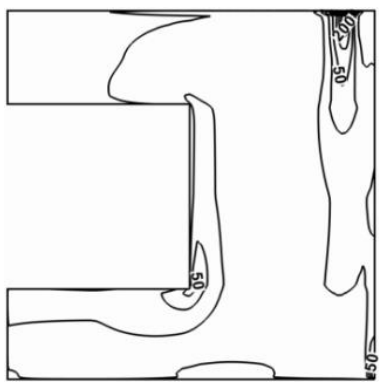

$H a=25$

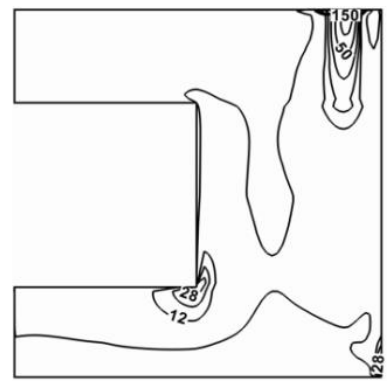

$H a=50$

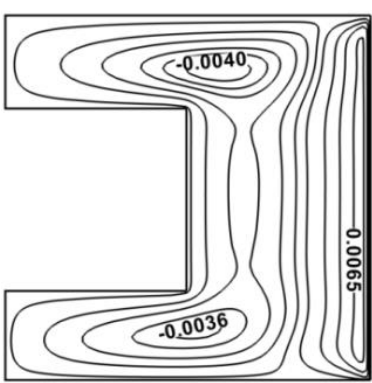

$H a=100$
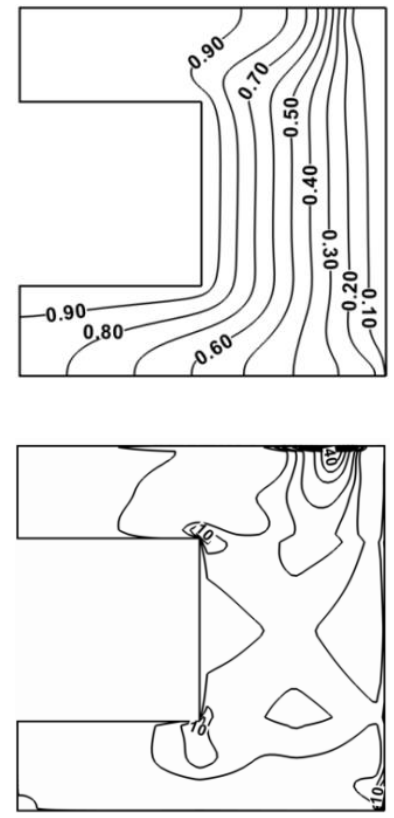

$H a=100$

Isentropic lines

Figure 7. Streamlines, isotherms and isentropic lines for various Ha number at Ri $=10$.

Without MHD effect $(H a=0)$, local Nusselt number shows high value on the $B C$ side than on the $A B$ and $C D$ sides of the hot wall when $R i=0.1$. It was because the $B C$ side of the hot wall and the cold movable wall were too close to each other. Consequently, heat is easily transported by counterclockwise recirculation cell induced by the moving wall. As $H a$ increases up to 100 , the local Nusselt number decreases around the $B$ point and the $B C$ side of the hot wall, whereas no significant variation is observed along the $A B$ and $C D$ sides.

When forced convection and natural convection are of the same order of magnitude (i.e., $R i=1$ ), the local Nusselt number shows a similar behavior as that relating to the case with $R i=0.1$ no matter $H a$ is, except that the maximum value at $B$ point is lower. As $R i$ number is increased from 1 to10, the local Nusselt number along the hot wall shows an inverse trend. In this case, the maximum value is observed at the $C$ point instead of the $B$ point due to the effect of clockwise cell generated by buoyancy force (Figure 7). In addition, as $\mathrm{Ha}$ number is increased up to100, the local Nusselt number is decreased alongside $A B C D$.
There is another key feature worth mentioning with regard to the peak of local Nusselt number at the $B$ and $C$ points for the three values of $R i$ number. In fact, at $R i=0.1$ and 1 the temperature distribution is almost equal to the thermal boundary condition (i.e. $\theta=1$ ) inside the lower corner of the cavity (Figures 5 and 6). This behavior resulted in a low temperature gradient between the hot side $C D$ and the fluid. Thus, low values of Nusselt number at the $C$ point and alongside $C D$ are observed. In the contrary, a reverse behavior is observed at $R i=10$ (Figure 7), leading to decrease local Nusselt number at the $B$ point and alongside $A B$. According to Figure 8 one can conclude that high heat transfer rate is occurred along the side $B C$ regardless of $R i$ and $H a$ numbers.

For comparison purpose, the $N u_{L}$ along the movable cold wall is also represented in Figure 8. Heat transfer shows an almost exponentially decreasing trend along the left cold wall. Heat is transferred from the heated sides (i.e. $A B C D)$ at highest rate near the lower left wall while a lower heat transfer rate is observed near the top of the left wall, no matter $R i$ and $H a$ numbers are. 

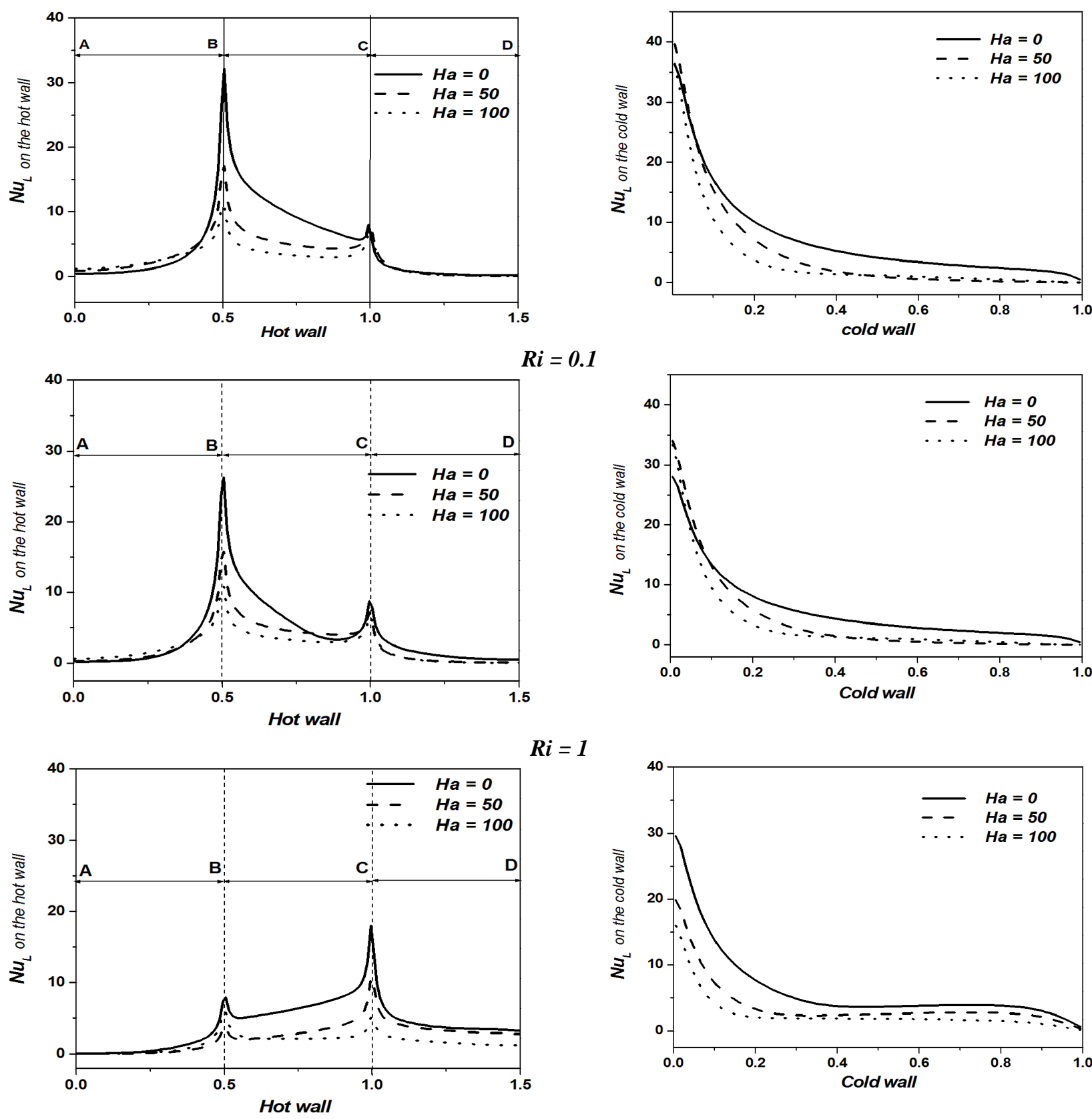

$R i=1$

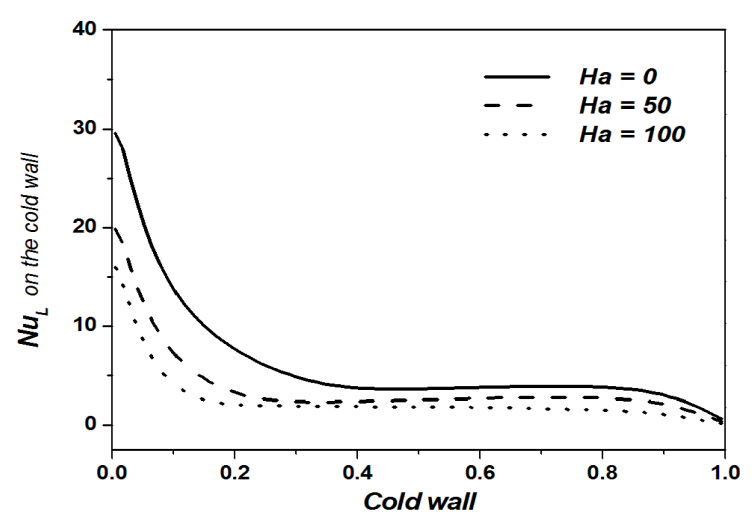

$R i=10$

Figure 8. Local Nusselt number along the hot wall (on the left) and along the cold wall (on the right) of the cavity for various values of Ri and Ha numbers.

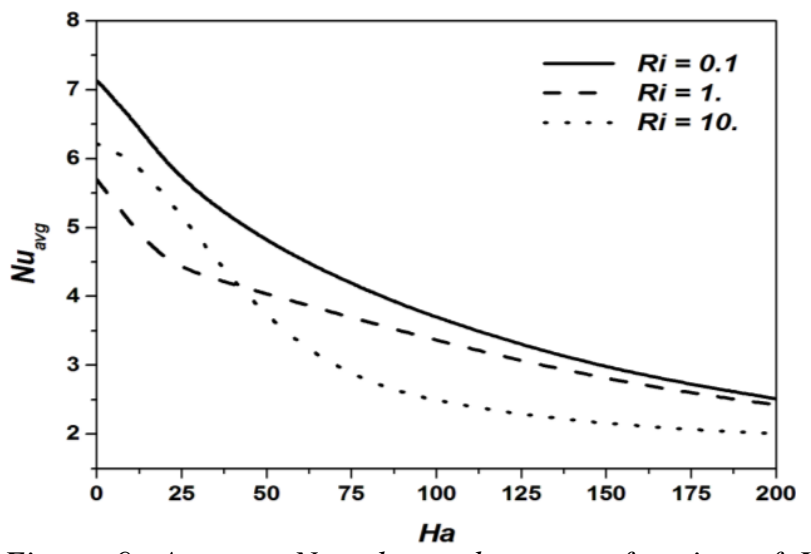

Figure 9. Average Nusselt number as a function of $\mathrm{Ha}$ number at $\alpha=0^{\circ}$ and $A R=0.5$.
Figure 9 illustrates the average Nusselt number variation $\left(N u_{\text {avg }}(\right.$ at the hot wall $)=N u_{\text {avg }}($ at the cold wall $)=$ $N u_{\text {avg }}$ ) as a function of the $H a$ number. This figure shows a reduction in $N u_{a v g}$ by increasing the $\mathrm{Ha}$ number regardless of the value of $R i$. It is also shown that the rate of decrease of $N u_{\text {avg }}$ corresponding to the case with $R i=0.1$ remains the lowest compared to the cases with $R i=1$ and 10 .

Moreover, it is observed that as $\mathrm{Ha}<50$, the curve relating to $R i=10$ overcomes that relating to $R i=1$, indicating that the rate of decrease of $N u_{\text {avg }}$ relating to the case with $R i=10$ is less than that relating to $R i=1$. Such behavior is due the fact that the case with $R i=10$, the heat cannot be well convected from the hot wall $A B C D$ to the right cold wall of the cavity due to the fact that the clockwise recirculation cell generated by the buoyancy force prevents the direct heat exchange.

The average Nusselt number variation as a function of $\mathrm{Ri}$ number is depicted in Figure 10 for various values of $\mathrm{Ha}$ number at $\alpha=0^{\circ}$ and $A R=0.5$. Without MHD effect, a 
sharp decrease of $N u_{\text {avg }}$ is observed until it reaches a minimum value at a given value of $R i$ number $(R i=1.5)$, thereafter, it increases monotonously. As $\mathrm{Ha}$ is increased to 50 and 100, the same behavior is observed as the case without MHD effect where, as expected, a less pronounced minimum and a significant decrease in $N u_{\text {avg }}$ are observed.

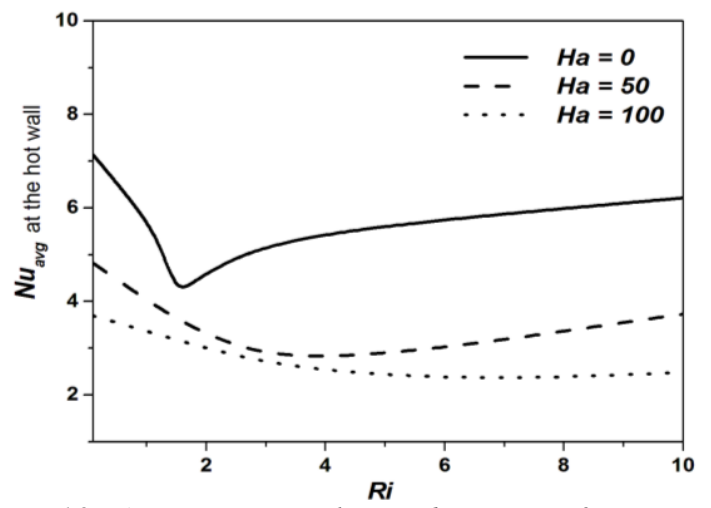

Figure 10. Average Nusselt number as a function of $R i$ number at $\alpha=0^{\circ}$ and $A R=0.5$.

The effect of $H a$ number on the total entropy generation $\left(S_{T}\right)$ and the average Bejan number for the cases of the forced convection and natural convection dominated mode, i.e., at $R i=0.1$ and 10, respectively is presented in Figure 11 for $\alpha=0^{\circ}$ and $A R=0.5$. The local entropy generation is decreased as $H a$ number increases for both values of $R i$ number as shown in Figure 11a. In addition, $\left(S_{T}\right)$ shows high values for $R i=0.1$ than that for $R i=10$ due to fact that the fluid friction is more pronounced in the case where the heat transfer is dominated by forced convection.

It is seen from Figure $11 \mathrm{~b}$ that the average Bejan number $\left(B e_{\text {avg }}\right)$ is decreased as $\mathrm{Ha}$ number increases up to 100 , thereafter it increases monotonously for both values of Richardson number $(R i=0.1$ and 10$)$. It is observed also that the $B e_{\text {avg }}$ is slightly decreased as $R i$ changed from 0.1 to 10. In addition, the value of $B e_{\text {avg }}$ is around the value one (1). Thus, the entropy generation related to the heat transfer irreversibility (HTI) is more important than the fluid friction entropy and the magnetic field entropy.

\subsection{Effect of the tilting angle of the cavity $(\alpha)$}

Figure 12 shows the effect of the tilting angle of the cavity on the local Nusselt number variation alongside $A B C D$ at $H a=0$ and $A R=0.5$ for $R i=0.1$ (forced convection dominant mode) and $R i=10$ (natural convection dominant mode).

In the forced convection dominated mode $(R i=0.1)$, the local Nusselt number at the hot wall is insensitive as to the variation of cavity inclination. In the natural convection dominated mode, it is found that the major changes occurring in the local Nusselt number are observed along the sides $B C$ and $C D$ as the cavity inclination takes the values $-45^{\circ}$ and $+45^{\circ}$ as it can be seen in Figure 12 (at $R i=$ 10).

Average Nusselt number $\left(N u_{\text {avg }}\right)$ as a function of $R i$ number is shown in Figure 13. It is observed that the trend of $N u_{\text {avg }}$ presents two distinct zones depending on $R i$ number: The first one corresponds to the forced convection dominated mode in which $N u_{\text {avg }}$ decreases steeply as $R i$ increases and reach a minimum that depends on the $R i$ number and the cavity inclination. Thus for $\alpha=0^{\circ},-45^{\circ}$ and $+45^{\circ}$, the corresponding $R i$ numbers are; 1.5, 2 and 2.8, respectively.

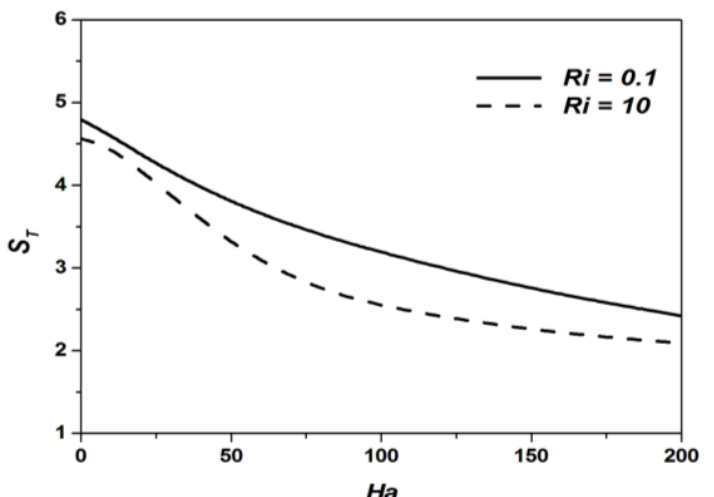

(a)

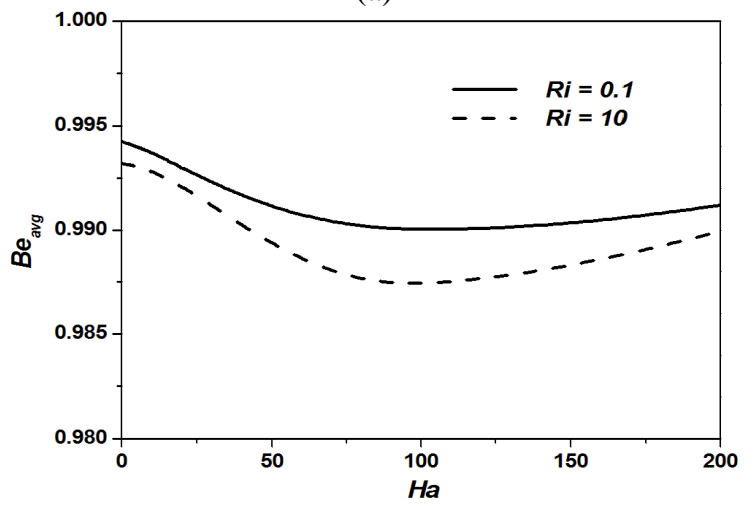

(b)

Figure 11. Total entropy generation (a) and average Bejan number (b) as a function of Ha number for R $i=0.1$ and 10 at $A R=0.5$ and $\alpha=0^{\circ}$.
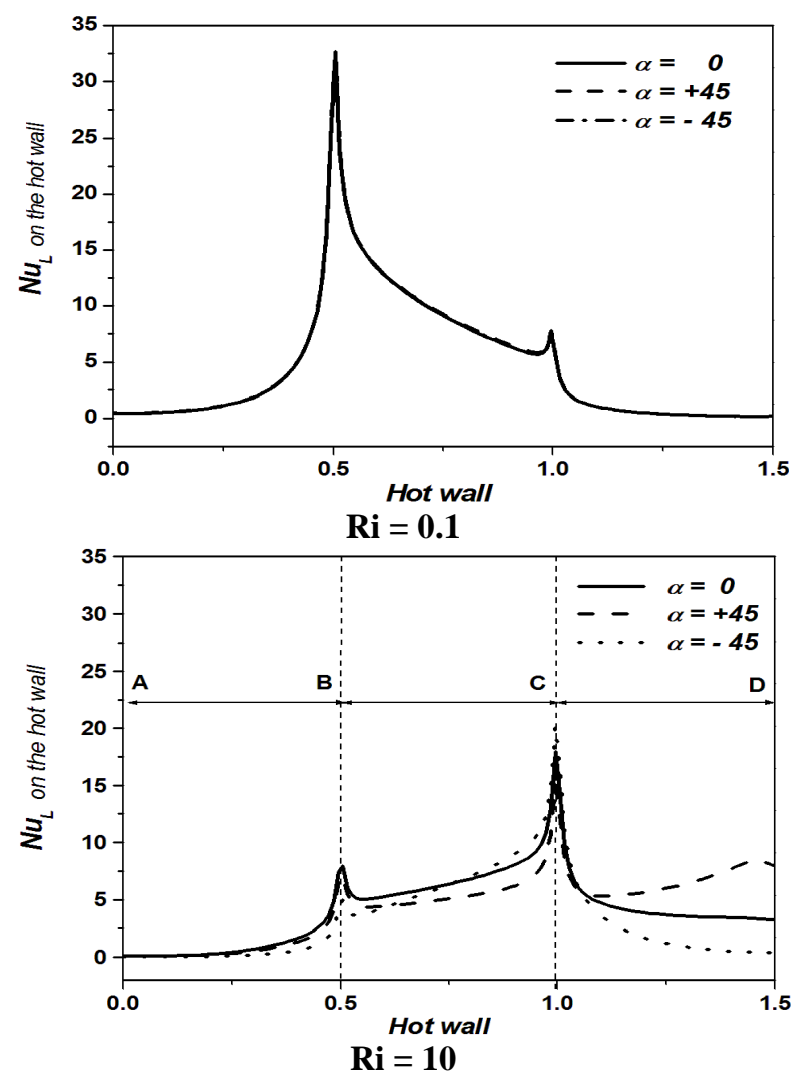

Figure 12. Local Nusselt number along inside hot walls for various values of tilting angle at $\mathrm{Ha}=0$ and $A R=0.5$.

The second zone to the natural convection dominated mode in which $N u_{\text {avg }}$ experience a monotonic increase with $R i$ regardless of the cavity inclination. This trend can be explained by the coupling effect of the clockwise cell 
generated by the buoyancy force and the counterclockwise one generated by moving lid. In addition, the same values of $N u_{\text {avg }}$ are observed when $R i<2$ for $\alpha=-45^{\circ}$ and $0^{\circ}$.

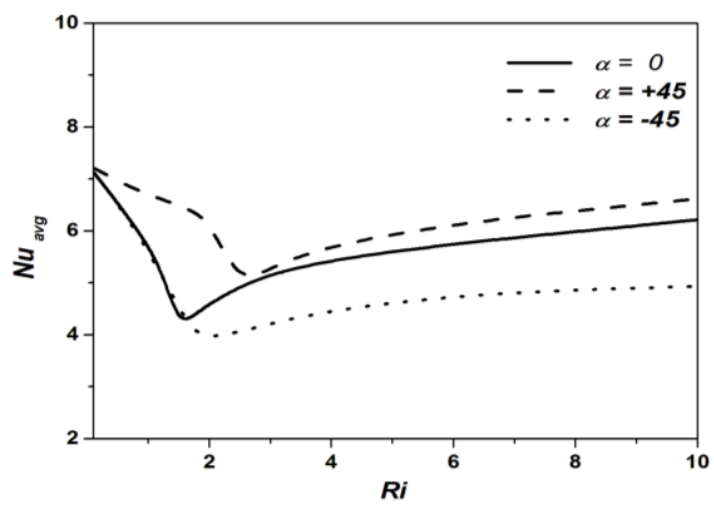

Figure 13. Average Nusselt number as a function of Ri for various tilting angle at $\mathrm{Ha}=0$ and $A R=0.5$.

Figure 14 illustrates the effect of the $\mathrm{Ha}$ number on $N u_{\text {avg }}$ for three values of cavity inclination angle $\left(0^{\circ},-45^{\circ}\right.$ and $+45^{\circ}$ ) at $R i=10$ and $A R=0.5$. A careful examination of this figure reveals that $N u_{\text {avg }}$ decreases monotonically towards an asymptotic value irrespective of $\alpha$ as $\mathrm{Ha}$ increases. This is due to the fact that as $\mathrm{Ha}$ increases, the Lorentz force increases in its turn leading to modify the velocity and pressure fields characteristics of the flow. Hence, heat transfer rate is decreased. In addition, $N u_{\text {avg }}$ is increased by changing of the cavity inclination angle from $45^{\circ}$ to $+45^{\circ}$ and shows the highest values for $\alpha=+45^{\circ}$ due to the fact that this position favors the increase of the strength of the clockwise circulating cell generated by the buoyancy force (which raises the fluid particles heated near the side $B C$ ). Consequently, the maximum stream function $\psi_{\max }$ passes from 0.019 for $\alpha=-45^{\circ}$ to 0.04 for $\alpha=+45^{\circ}$. As a result, the heat transfer rate is increased.

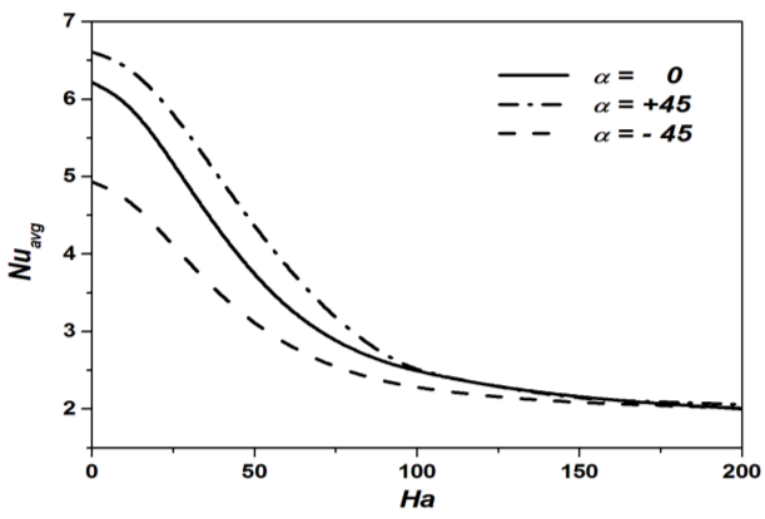

Figure 14. Average Nusselt number as a function of Ha for various values of tilting angle at $R i=10$ and $A R=0.5$.

The effect of tilting angle of the cavity varied from $\alpha=$ $45^{\circ}$ to $+45^{\circ}$, with an increment of $15^{\circ}$ on $N u_{\text {avg }}$ is shown in Figure 15 . In the case of forced convection dominated mode $(R i=0.1)$, no significant change of $N u_{\text {avg }}$ is observed by varying $\alpha$ from $-45^{\circ}$ to $+45^{\circ}$, while in the case of $R i=1$ and 10, two different trends of $N u_{\text {avg }}$ are observed. The average Nusselt number $\left(N u_{\text {avg }}\right)$ starts decreasing with the increase of $\alpha$ until it reaches a minimum value, then, it increases almost linearly with further increases in $\alpha$ for $R i=1$. In the buoyancy dominated mode $(R i=10)$, the $N u_{\text {avg }}$ increases monotonously by increasing the inclination angle $\alpha$ from - $45^{\circ}$ to $+45^{\circ}$. It is also observed that in this case (buoyancy dominated mode) when the tilting angle of the cavity below $-30^{\circ}$ leads to disadvantages the heat transfer rate inside the C-shaped cavity.

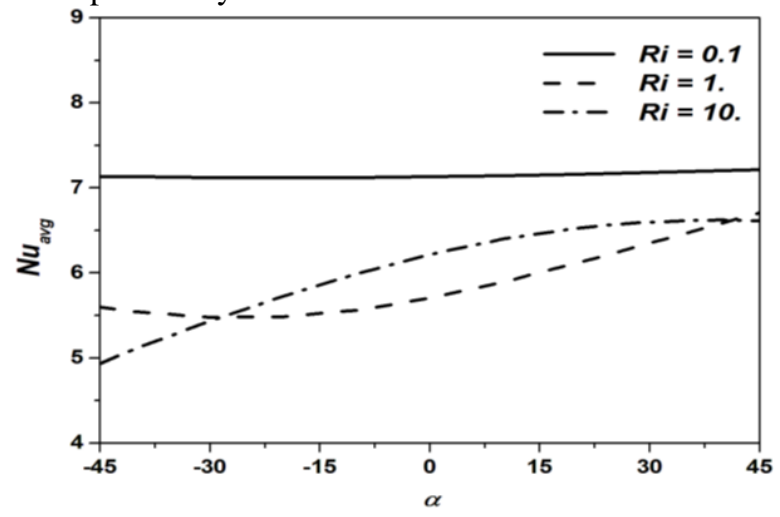

Figure 15. Average Nusselt number as a function angle for various values of Ri number at $H a=0$ and $A R=0.5$.

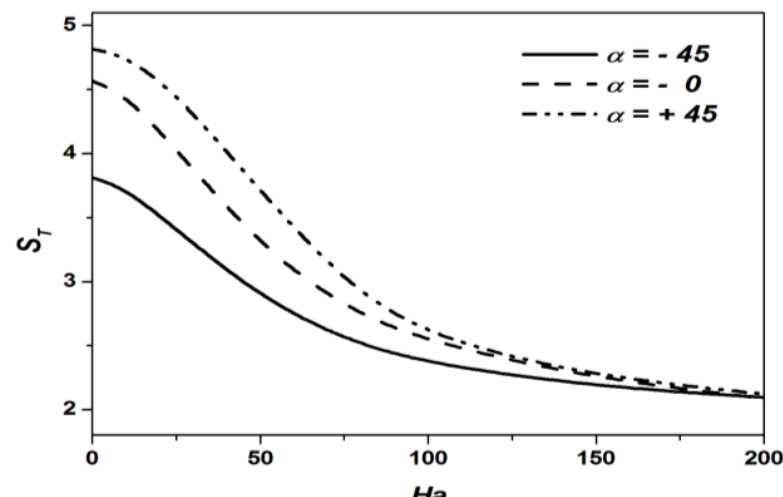

(a)

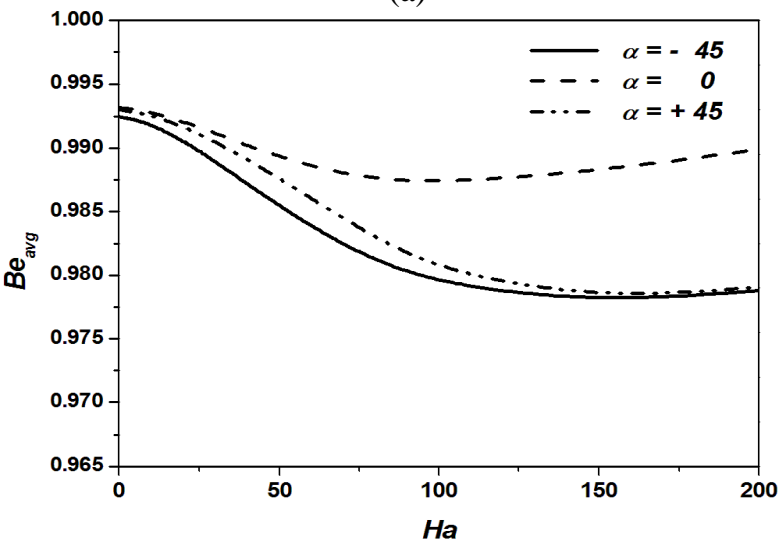

(b)

Figure 16. Total entropy generation (a) and average Bejan number (b) as a function of Ha number for various values of tilting angle at $R i=10$ and $A R=0.5$.

Figure 16 presents the effect of $\mathrm{Ha}$ number on the total entropy generation and the average Bejan number for a wide range of inclination angles in the case of natural convection dominated mode $(R i=10)$ at $A R=0.5$. As can be seen from Figure 16a, the total entropy generation decreases monotonically till an asymptotic value at high value of $H a$ number no matter $\alpha$ is. Moreover, without MHD effect the total entropy generation increases as the position of cavity changed from $-45^{\circ}$ to $+45^{\circ}$ and shows the highest values at $\alpha=+45^{\circ}$. It is also observed that the heat transfer irreversibility is the major contribution in the total entropy generation as $\mathrm{Ha}$ increases. This is due to the fact 
that the fluid flow becomes practically immobile except along the moving wall.

The average Bejan number $\left(B e_{\text {avg }}\right)$ is decreased as $\mathrm{Ha}$ number increases for $\alpha=-45^{\circ}$ and $+45^{\circ}$, whereas for $\alpha=0^{\circ}$ the variation of $B e_{\text {avg }}$ is very weak, Figure $16 \mathrm{~b}$. Moreover, as $\mathrm{Ha}$ increases, the value of $B e_{\text {avg }}$ is approximately equal to 1 for all values of tilting angle, indicating that the entropy generation is dominated by heat transfer irreversibility (HTI).

\subsection{Effect of aspect ratio (AR)}

The effect of the aspect ratio on the streamlines for three values of $R i$ number $\left(0.1,1\right.$ and 10) at $H a=0$ and $\alpha=0^{\circ}$ in Figure 17. It is worth mentioning to note that as the aspect ratio increases the heated surface of the $\mathrm{C}$-shaped cavity (i. e., $A B C D$ side) increases while the enlargement of the inner space of the cavity decreases. For $R i=0.1$, a single circulation cell in clockwise direction generated by lid- driven wall is observed regardless $A R$ values. As $R i$ is increased to 1 , secondary recirculation cell adjacent to the hot side $B C$ is observed occupying almost the same internal space of the cavity as that generated by forced convection at $A R=0.3$. It is also observed that the strength secondary recirculation cell is lower than that generated by forced convection. By increasing the aspect ratio to 0.5 and 0.7 , the secondary cell splits into two cells and which are pushed to the top and bottom corners of the cavity under the effect of the primary recirculation cell. As Richardson in increased to 10 (natural convection dominated regime), the intensity and extent of the secondary anti-clockwise cell generated by buoyancy force increase to the detriment of the cell generated by moving lid for $A R=0.3$ and 0.5 . For $A R=0.7$, the two cells occupy almost the same space comprised between the lid wall and the hot side $B C$ and show the same strength, while the fluid located at the top and bottom corners of the cavity is almost stagnant.
$A R=0.3$

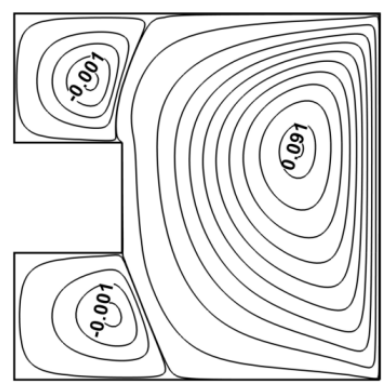

$A R=0.5$

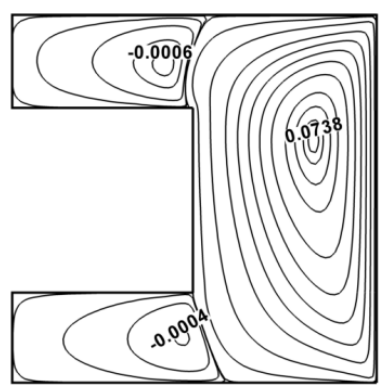

$A R=0.7$

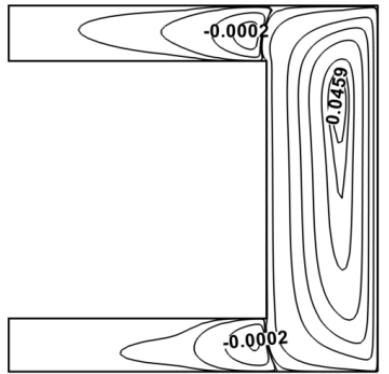

$R i=0.1$
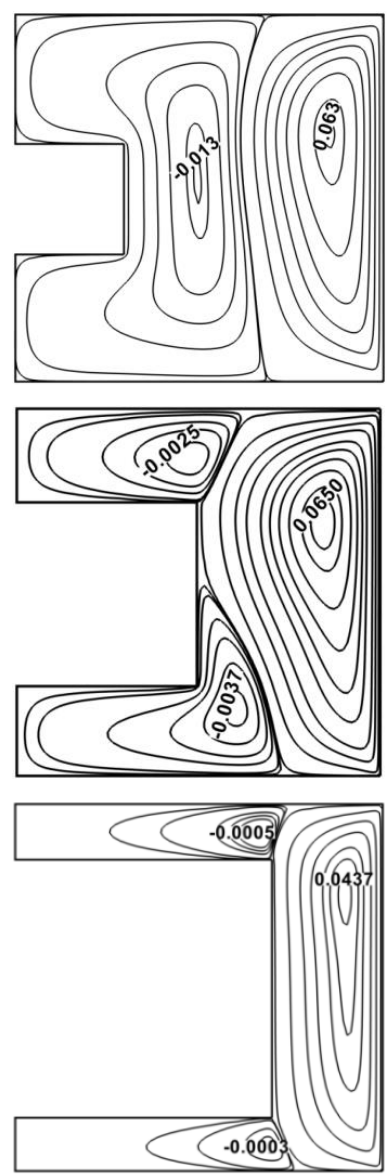

$R i=1$
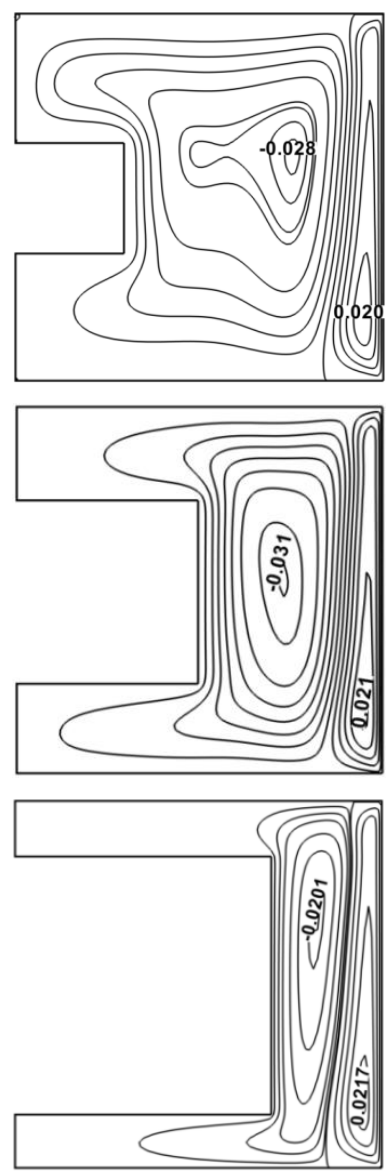

$R i=10$

Figure 17. Streamlines for various Richardson numbers and different AR at $H a=0$ and $\alpha=0^{\circ}$.

The effect of $R i$ number on the average Nusselt number for different values of aspect ratio $(A R=0.3,0.5$ and 0.7$)$ is illustrated in Figure 18 at $\mathrm{Ha}=0$ and $\alpha=0^{\circ}$. Starting from a given value which increases with the aspect ratio, a sharp decrease in $N u_{a v g}$ to a minimum value is observed regardless of aspect ratio value as $R i$ number increases. It is also observed that the value of the $R i$ number where the minimum of the average heat transfer rate is attained increases by increasing the aspect ratio. Also, the result showed that beyond this minimum, $N u_{\text {avg }}$ increases with $R i$ number and the curves relating to the three aspect ratio values get close to each other. In addition, by increasing the aspect ratio of the cavity the minimum of the heat transfer rate disappear as shown for the case with $A R=0.7$.

Figures $19 \mathrm{a}-\mathrm{c}$ present the variation of $N u_{\text {avg }}$ as a function of $\mathrm{Ha}$ number for different values of aspect ratio at $\alpha=$ $45^{\circ}, 0^{\circ}$ and $+45^{\circ}$ and $R i=10$. It can be seen that up to $H a=$ 50 , no significant change is observed in $N u_{\text {avg }}$ for $\alpha=+45^{\circ}$ and $0^{\circ}$. Beyond this value, $N u_{\text {avg }}$ shows different rates of decreases depending on the value of aspect ratio of the cavity. The results for $\alpha=-45^{\circ}$ show significant spacing between $N u_{\text {avg }}$ curves whatever $H a$ number. 


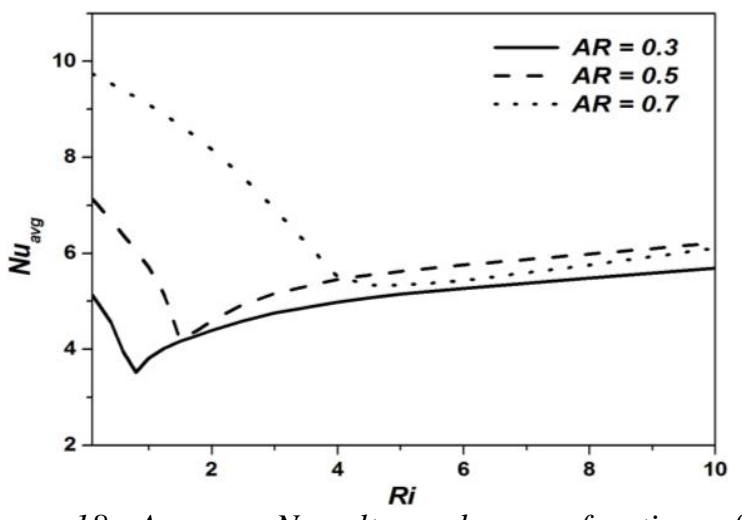

Figure 18. Average Nusselt number as function of $R i$ number for various $A R(0.3,0.5$ and 0.7) at $\mathrm{Ha}=0$ and $\alpha=$ $0^{\circ}$.

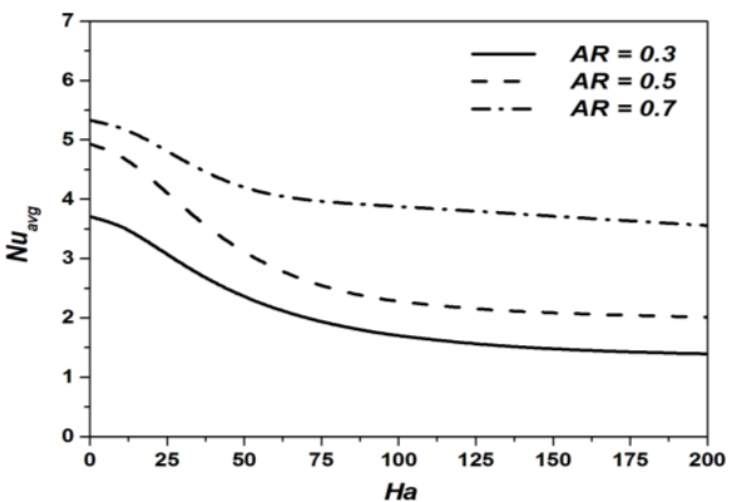

(a)

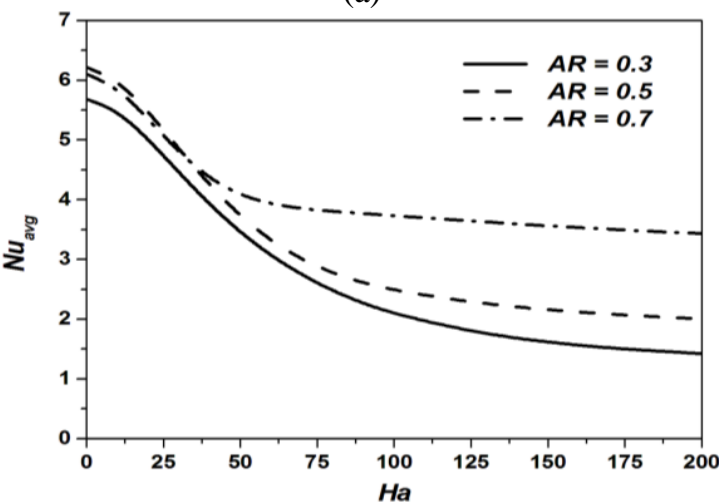

(b)

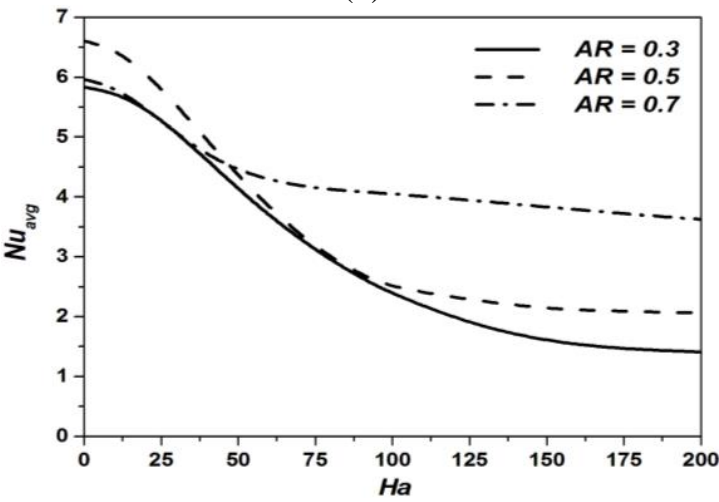

(c)

Figure 19. Average Nusselt number as function of Hartmann number for various $A R$ at different tilting angles; (a) $\alpha=-45^{\circ}$, (b) $\alpha=0^{\circ}$ and (c) $\alpha=+45^{\circ}$ at $R i=10$.

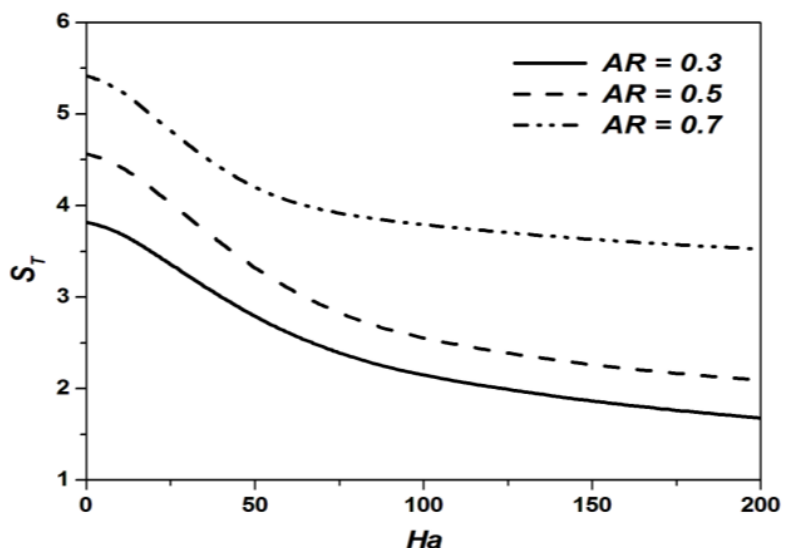

(a)

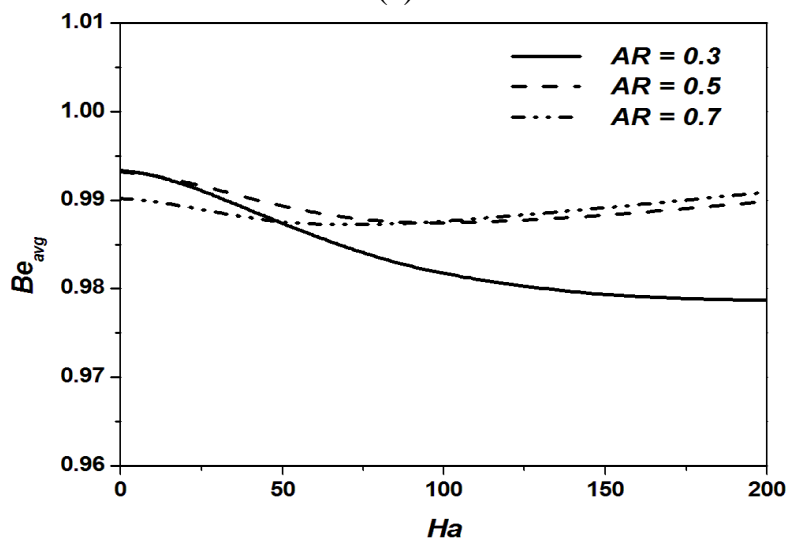

(b)

Figure 20. Total entropy generation (a) and average Bejan number (b) as a function of Ha number for various values of aspect ratio angle at $R i=10$ and $\alpha=0^{\circ}$.

It is also observed in Figures 19a-c that as the aspect ratio of the cavity increases, the effect of $\mathrm{Ha}$ number on $N u_{\text {avg }}$ goes down. This is due to the fact that when the value of $\mathrm{Ha}$ number is greater that $70(\mathrm{Ha}>70)$, the heat transfer mechanism tends to be conductive state.

It is worth to recall that, the increase in the aspect ratio of the cavity results in an increase of the surface of the hot wall $(A B C D)$ and a decrease in the distance between the hot wall and the movable cold wall which contributes to the improvement of heat transfer.

Figure 20 represents the variation of the total entropy generation (Figure 20a) and the average Bejan number (Figure 20b) as a function of $\mathrm{Ha}$ number for various values of the aspect ratio $(A R=0.3,0.5$ and 0.7$)$ at $R i=10$ and $\alpha=$ $0^{\circ}$.

As can be seen from Figure 20a, a decreasing of total entropy generation as $H a$ number increases is observed irrespective of $A R$ due to the fact that the fluid friction is decreased as velocity of fluid flow decreases. Besides, the value of total entropy generation is increased as $A R$ increases due to a corresponding increase in the heat transfer rate.

A monotone decrease of the average Bejan number $\left(B e_{\text {avg }}\right)$ is observed as $\mathrm{Ha}$ number increases for the case of $A R=0.3$, whereas for $A R=0.5$ and 0.7 , the variation of $B e_{\text {avg }}$ is very weak as shown in Figure 20b. Moreover, the value of $B e_{\text {avg }}$ is approximately equal to 1 as $\mathrm{Ha}$ increases for all values of aspect ratio of the $\mathrm{C}$-shaped cavity $(A R)$, indicating the dominance of entropy generation related to the heat transfer irreversibility.

\section{Conclusion}

Vol. 24 (No. 4) / 13 
A numerical investigation of MHD mixed convection and entropy generation under the influence of magnetic field inside a C-shaped cavity filled by an electrically conducting fluid is presented. The effects of Richardson number, Hartmann number, aspect ratio and tilting angle of cavity on the convection heat transfer rate and entropy generation are discussed.

Based on the above results, the main conclusions of this study are as follows:

1- The heat transfer rate is found to be enhanced in the forced convection dominated mode $(R i=0.1)$ irrespective of $H a, \alpha$ and $A R$.

2- The total entropy generation increases with an increasing of $A R$ and $\alpha$ and decreases as $H a$ and $R i$ numbers increase.

3- The total entropy generation is predominantly due to heat transfer irreversibility no matter $A R, \alpha, R i$ and $H a$ are.

4- In the case of forced convection dominated mode $(R i=$ 0.1 ), the effect of tilting angle is negligible, contrary to the case related to $R i=1$ and 10 where its effect is significant.

5- At low values of $\mathrm{Ha}$ number, the effect of tilting angle on heat transfer is more pronounced, whereas at high values, no significant effect is observed.

6- The effect of $A R$ is noticeable on heat transfer rate in the case of forced convection dominated mode. In the case of natural convection dominated mode, no enhancement of heat transfer rate is observed by varying $A R$.

7- At high values of $H a$ number, the heat transfer is mainly done by conduction regardless of $A R, R i$ and $\alpha$.

\section{Nomenclature}

\begin{tabular}{|c|c|}
\hline & \\
\hline$A R$ & aspect ratio of $\mathrm{C}$ shape cavity \\
\hline$B e_{\text {avg }}$ & average Bejan number \\
\hline$B_{0}$ & magnetic field $(\mathrm{T})$ \\
\hline$g$ & gravitational acceleration $\left(\mathrm{m} . \mathrm{s}^{-2}\right)$ \\
\hline$G r$ & Grashof number \\
\hline$H$ & cavity height (m) \\
\hline $\mathrm{Ha}$ & Hartmann number \\
\hline$L$ & cavity length (m) \\
\hline$N u_{L}$ & local Nusselt number \\
\hline$N u_{\text {avg }}$ & average Nusselt number \\
\hline$p$ & pressure $(\mathrm{Pa})$ \\
\hline$P$ & dimensionless pressure \\
\hline $\operatorname{Pr}$ & Prandtl number \\
\hline $\operatorname{Re}$ & Reynolds number \\
\hline$R i$ & Richardson number \\
\hline$S$ & Dimensionless entropy generation \\
\hline$T$ & temperature $(\mathrm{K})$ \\
\hline$u$ & horizontal velocity $(\mathrm{m} / \mathrm{s})$ \\
\hline$U$ & dimensionless horizontal velocity \\
\hline$v$ & vertical velocity $(\mathrm{m} / \mathrm{s})$ \\
\hline$V$ & dimensionless vertical velocity \\
\hline$x, y$ & Cartesian coordinates $(\mathrm{m})$ \\
\hline$X, Y$ & dimensionless Cartesian coordinates \\
\hline Greek & ymbols \\
\hline$\alpha_{f}$ & thermal diffusivity $\left(\mathrm{m}^{2} . \mathrm{s}^{-1}\right)$ \\
\hline$\alpha$ & tilting angle of the cavity $\left(^{\circ}\right)$ \\
\hline$\beta$ & thermal expansion coefficient $\left(\mathrm{K}^{-1}\right)$ \\
\hline$\mu$ & dynamic viscosity $\left(\mathrm{kg} \cdot \mathrm{m}^{-1} \cdot \mathrm{s}^{-1}\right)$ \\
\hline$v$ & kinematic viscosity $\left(\mathrm{m}^{2} . \mathrm{s}^{-1}\right)$ \\
\hline$\sigma$ & electrical conductivity $(1 / \Omega . \mathrm{m})$ \\
\hline
\end{tabular}

$\begin{array}{ll}\chi_{i} & \text { irreversibility coefficient }(\mathrm{i}=1,2) \\ \theta & \text { dimensionless temperature } \\ \rho & \text { density }\left(\mathrm{kg} / \mathrm{m}^{3}\right) \\ \text { Subscripts } \\ \text { avg } \quad \text { average } \\ c & \text { cold } \\ L & \text { local } \\ h & \text { hot } \\ M a g & \text { magnetic } \\ T h & \text { thermal } \\ V & \text { viscous } \\ T & \text { total }\end{array}$

\section{References:}

[1] K. H. Hameed, H. A. Farooq, M. Hatami, D. Jing, "Effect of two baffles on MHD natural convection in U-shape superposed by solid nanoparticle having different shapes," Journal of Applied and Computational Mechanics, 6, 1200-1209, 2020.

[2] Y. Menni, A. Azzi, A. J. Chamkha, "A review of solar energy collectors: models and applications," Journal of Applied and Computational Mechanics, 4, 375-401, 2018.

[3] G.M. Oreper, J. Szekely, "The effect of an externally imposed magnetic field on buoyancy driven flow in a rectangular cavity," Journal of Crystal Growth, 64, 505-515, 1983.

[4] H. Ozoe, E. Maruo, "Magnetic and gravitational natural convection of melt silicon-tow-dimensional numerical computations for the rate of heat transfer," JSME International Journal, 30,774-784, 1987.

[5] J. P. Garandet, T. Alboussiere, "Buoyancy driven convection in a rectangular enclosure with a transverse magnetic field," Int. J. Heat Mass Transfer," 35, 741-748, 1992.

[6] K. Okada, H. Ozoe, "Experimental heat transfer rates of natural convection of molten gallium suppressed under an external magnetic field in either the X,Y, or Z direction," Journal of Heat Transfer, 114, 107-114, 1992.

[7] A. F. Emery, "The effect of a magnetic field upon the free convection of a conducting fluid," Journal of Heat Transfer, 85, 119-124, 1963.

[8] M. Venkatachalappa, C. K. Subbaraya, "Natural convection in a rectangular enclosure in the presence of a magnetic field with uniform heat flux from the side walls," Acta Mechanica, 96, 13-26, 1993.

[9] S. Alchaar, P. Vasseur, E. Bilgen, "Natural convection heat transfer in a rectangular enclosure with a transverse magnetic field," Journal of Heat Transfer, 117, 668-673, 1995.

[10] N. Rudraiah, R. M. Barron, M. Venkatachalappa, C. K. Subbaraya, "Effect of a magnetic field on free convection in a rectangular enclosure," Int. J. Engng. Sci., 33, 1075-1084, 1995.

[11] N.M. Al-Najem, K.M. Khanafer, M.M. El-Refaee, "Numerical study of laminar natural convection in tilted enclosure with transverse magnetic field," International Journal of Numerical Methods for Heat \& Fluid Flow, 8 , 651-672, 1998.

[12] M. C. Ece, E. Büyük, "Natural-convection flow under a magnetic field in an inclined rectangular enclosure heated and cooled on adjacent walls," 
Fluid Dynamics Research, 38, 564-590, 2006.

[13] M. Sathiyamoorthy, A. J. Chamkha, "Natural convection flow under magnetic field in a square cavity for uniformly (or) linearly heated adjacent walls," International Journal of Numerical Methods for Heat \& Fluid Flow, 22, 677-698, 2012.

[14] M. Sathiyamoorthy, A. J. Chamkha, "Effect of magnetic field on natural convection flow in a liquid gallium filled square cavity for linearly heated side wall(s)," International Journal of Thermal Sciences, 49, 1856-1865, 2010.

[15] M. Bhuvaneswari, S. Sivasankaran Y. J Kim, "Magneto-convection in a square enclosure with sinusoidal temperature distributions on both side walls," Numerical Heat Transfer, Part A, 59,167184, 2011.

[16] M. Hasanuzzaman, H. F. Öztop, M.M. Rahman, N.A. Rahim, R. Saidur Y. Varol, "Magnetohydrodynamic natural convection in trapezoidal cavities," Int. Communications in Heat and Mass Transfer, 39, 1384-1394,2012.

[17] M.S. Hossain, M.A. Alim, "MHD free convection within trapezoidal cavity with non-uniformly heated bottom wall," International Journal of Heat and Mass Transfer, 69, 327-336, 2014.

[18] M. Sheikholeslami, I. Hashim, S. Soleimani, "Numerical investigation of the effect of magnetic field on natural convection in a curved-shape enclosure," Mathematical Problems in Engineering, 2013, 1-10, 2013.

[19] A. Sahi, D. Sadaoui, N. Sadoun A. Djerrada, "Effects of magnetic field on natural convection heat transfer in a T-shaped cavity," Mechanics \& Industry, 18, 1-14, 2017.

[20] A. Jahanbakhshi, A. A. Nadooshan, M. Bayareh, "Magnetic field effects on natural convection flow of a non-Newtonian fluid in an L-shaped enclosure," Journal of Thermal Analysis and Calorimetry, 133, 1-10, 2018.

[21] A. A. Mohamad, I. Sezai, "Natural convection In Cshaped thermosyphone," Numerical Heat Transfer, Part A, 32, 311-323, 1997.

[22] M. Izadi, R. Mohebbi, A. Chamkha, "Effects of cavity and heat source aspect ratios on natural convection of a nanofluid in a $\mathrm{C}$-shaped cavity using Lattice Boltzmann method," International Journal of Numerical Methods for Heat \& Fluid Flow, 28, 1930-1955, 2018.

[23] M. A. Mansour, M. A. Bakeir, A. Chamkha, "Natural convection inside a C-shaped nanofluid-filled enclosure with localized heat sources," International Journal of Numerical Methods for Heat \& Fluid Flow, 24, 1954 - 1978, 2014.

[24] A. Yadollahi, A. Khalesidoost, A. Kasaeipoor, M. Hatami, D. Jing, "Physical investigation on silver- water nanofluid natural convection for an F-shaped cavity under the magnetic field effects," Eur. Phys. J. Plus, 132, 340-3503, 2017.

[25] B. Ghasemi, "Magnetohydrodynamic natural convection of nanofluids in U-shaped enclosures," Numerical Heat Transfer, Part A, 63, 473-487, 2013.

[26] M. Bayareh, M. A. Kianfar, A. Kasaeipoor, "Mixed convection heat transfer of water-alumina nanofluid in an inclined and baffled C-shaped enclosure," Journal of Heat and Mass Transfer Research, 5, 129-138, 2018.

[27] S.M. Shavik, M.N. Hassan, A.K.M. Morshed, M.Q. Islam, "Natural convection and entropy generation in a square inclined cavity with differetially heated vertical walls," Procedia Engineering, 90, 557-562, 2014.

[28] S. Parvin, A. Chamkha, "An analysis on free convection flow, heat transfer and entropy generation in an odd-shaped cavity filled with nanofluid," International Communications in Heat and Mass Transfer, 54, 8-17, 2014.

[29] A. Chamkha, F. Selimefendigil, H. Oztop, "MHD mixed convection and entropy generation in a liddriven triangular cavity for various electrical conductivity models," Entropy, 20, 1-17, 2018.

[30] C. C. Cho, "Heat transfer and entropy generation of mixed convection flow in $\mathrm{Cu}$-water nanofluid-filled lid-driven cavity with wavy surface," International Journal of Heat and Mass Transfer, 119, 163-174, 2018.

[31] H. Khakrah, P. Hooshmand, D. Ross, M. Jamshidian, "Numerical analysis of free convection and entropy generation in a cavity using compact finite-difference lattice Boltzmann method," International Journal of Numerical Methods for Heat \& Fluid Flow, 30, 977-995, 2020.

[32] S.V. Patankar, Numerical Heat Transfer and Fluid Flow, Hemisphere, Washington, New York, 1980.

[33] R. Iwatsu, J. M. Hyun, K. Kuwahara, "Liquid mixed convection in a driven cavity with a stable vertical temperature gradient," Int. J. Heat Mass Transfer, 36, 1601-1608, 1993.

[34] S. Mojumder, S. Saha, S. Saha, M.A.H. Mamun, "Effect of magnetic field on natural convection in a C-shaped cavity filled with ferrofluid," Procedia Engineering, 105, 96-104, 2015. 